\title{
miR-193b exhibits mutual interaction with MYC, and suppresses growth and metastasis of osteosarcoma
}

\author{
JINJIAN GAO ${ }^{1,2^{*}}$, SAI MA $^{3 *}$, FAN YANG $^{4}, \mathrm{XU} \mathrm{CHEN}^{4}$, WEI WANG ${ }^{4}$, \\ JIANPING ZHANG ${ }^{5}$, YUFANG $\mathrm{LI}^{4}$, TAO WANG ${ }^{4,6}$ and LEQUN SHAN ${ }^{1}$ \\ ${ }^{1}$ Department of Spine Surgery, Honghui Hospital, Xi'an Jiaotong University, Xi'an, Shaanxi 710054; ${ }^{2}$ Department of \\ Orthopedics, Tangdu Hospital, The Fourth Military Medical University, Xi'an, Shaanxi 710038 ; ${ }^{3}$ Department of \\ Prosthodontics, School of Stomatology; ${ }^{4}$ Department of Immunology, The Fourth Military Medical University, \\ Xi'an, Shaanxi 710032; ${ }^{5}$ Department of Orthopedics, Kunming General Hospital of Chengdu Military \\ Command, Kunming, Yunnan 650032; ${ }^{6}$ Department of Medical Genetics and Developmental \\ Biology, The Fourth Military Medical University, Xi'an, Shaanxi 710032, P.R. China
}

Received March 10, 2019; Accepted February 19, 2020

DOI: 10.3892/or.2020.7601

\begin{abstract}
Emerging evidence has indicated that microRNAs (miRs) are involved in the malignant behavior of cancer. The present study explored the role of miR-193b in the development and metastasis of osteosarcoma. Compared with F4 osteosarcoma cells, which have a relatively low metastatic potential, highly metastatic F5M2 cells exhibited a lower expression of miR-193b. Furthermore, miR-193b exerted negative effects on cell proliferation, colony formation, cell cycle progression, migration and invasion, and induced apoptosis. In vivo studies revealed negative influences of miR-193b on tumorigenesis and metastasis. The tumor-suppressive role of miR-193b was achieved by targeting KRAS and stathmin 1 (STMN1). Notably, overexpression of KRAS and STMN1 attenuated the miR-193b-induced inhibition of malignant behaviors. There was a double-negative regulatory loop between MYC and miR-193b, with MYC inhibiting miR-193b expression by directly binding to its promoter region and miR-193b negatively influencing MYC expression indirectly through some unknown mechanism. Collectively, these findings indicated that miR-193b may serve a tumor suppressive
\end{abstract}

Correspondence to: Professor Lequn Shan, Department of Spine Surgery, Honghui Hospital, Xi'an Jiaotong University, 555 YouYi East Road, Xi'an, Shaanxi 710054, P.R. China

E-mail: drshanlq@163.com

Professor Tao Wang, Department of Medical Genetics and Developmental Biology, The Fourth Military Medical University, 127 Changlexi Road, Xi'an, Shaanxi 710032, P.R. China

E-mail: wangt@fmmu.edu.cn

*Contributed equally

Key words: osteosarcoma, microRNA, carcinogenesis, oncogene, cell proliferation role in osteosarcoma by targeting KRAS and STMN1. The double-negative regulatory loop between MYC and miR-193b may contribute to the sustained upregulation of $\mathrm{MYC}$, the downregulation of miR-193b, and to the subsequently enhanced expression of KRAS and STMN1, which may eventually lead to the development and metastasis of osteosarcoma.

\section{Introduction}

From the 1960s to the 1980s, survival rates for patients with osteosarcoma significantly increased due to neoadjuvant and postsurgery chemotherapy (1). However, the outcome remains poor for the majority of patients with metastatic or recurrent osteosarcoma (2). Therefore, novel insights into the mechanisms underlying tumorigenesis and metastasis are urgently required to further improve overall survival in patients with osteosarcoma.

MicroRNAs (miRNAs/miRs) have important roles in the initiation and development of tumors $(3,4)$. Various miRNAs, which have been reported to act as either tumor promoters or tumor suppressors, have been identified in osteosarcoma (5-7). In our previous study, the miRNA expression spectrum of two osteosarcoma cell lines with different metastatic potentials was compared and a group of differentially expressed miRNAs was identified (8). Among the identified miRNAs, miR-193b revealed a $\sim 2$-fold difference between the high metastatic subline (F5M2) and the low metastatic subline (F4). Although the tumor-suppressive role of miR-193b has been demonstrated in several types of cancer (9-13), the involvement of miR-193b in osteosarcoma has rarely been discussed. Therefore, the purpose of the present study was to investigate the effects of miR-193b on the malignant behaviors of osteosarcoma, and to clarify its underlying mechanism by exploring its upstream regulator and downstream targets. KRAS is one of the front-line sensors that initiate the activation of an array of signaling molecules, thus affecting cell differentiation, growth, chemotaxis and apoptosis (14). Stathmin 1 (STMN1) regulates cell division by destabilizing microtubules in a 
phosphorylation-dependent manner, and acts as an important regulator of cell cycle progression, proliferation, invasion and survival (15). KRAS and STMN1 have been predicted as downstream effectors of miR-193b, and they have been reported to be upregulated in several human tumors, including osteosarcoma $(16,17)$. Thus, the present study focused on KRAS and STMN1 as downstream effectors of miR-193b to clarify the mechanism by which miR-193b may modulate malignant behaviors of osteosarcoma. As for the upstream regulator of miR-193b, this study focused on the well-recognized oncogene MYC, since it may be able to bind to the promoter region of miR-193b as predicted by bioinformatics tools (18).

The present study reported that miR-193b suppressed the malignant behaviors of osteosarcoma cells through the direct inhibition of its targets; namely, KRAS and STMN1. In addition, miR-193b and MYC exerted mutual suppressive effects against each other. Such mutual suppressive effects may lead to sustained MYC activation, miR-193b inhibition, and the eventual overexpression of KRAS and STMN1.

\section{Materials and methods}

Cell culture. F4 and F5M2 osteosarcoma cell lines with different metastatic potential, screened and established from the human osteosarcoma cell line SOSP-9607, were acquired from the Department of Orthopedics, Tangdu Hospital, Fourth Military Medical University (Xi'an, China) (8). 293A cells were purchased from the Institutes for Biological Sciences at the Chinese Academy of Sciences. Osteosarcoma cells and 293A cells were separately cultured in RPMI (HyClone; GE Healthcare Life Sciences) or DMEM (Gibco; Thermo Fisher Scientific, Inc.), respectively, supplemented with $10 \%$ fetal bovine serum (Gibco; Thermo Fisher Sciences, Inc.) at $37^{\circ} \mathrm{C}$ in a humidified incubator with $5 \% \mathrm{CO}_{2}$.

Oligonucleotides and plasmids. Synthetic miR-193b mimic, miR-193b inhibitor, negative controls (NC and inhibitor NC), silencer Select Negative Control (siNC), and KRAS, STMN1 and MYC small interfering RNAs (siRNAs) were obtained from Shanghai GenePharma Co., Ltd. Lentiviral particles containing miR-193b (LV-193b), miR-193b inhibitor (LV-193b inhibitor) and negative controls (LV-NC and LV-inhibitor-NC) were purchased from GeneCopoeia, Inc. The coding sequences of KRAS and STMN1 were PCR-amplified from F5M2 cell cDNA using primers listed in Table I and were cloned into the $B g$ III and XhoI sites of pMSCV (Addgene), yielding the pMSCV-KRAS and pMSCV-STMN1 overexpression vectors (19); empty pMSCV was used as the control. MYC overexpression vector and its control vector ( $\mathrm{pBabe-MYC}$ and pBabe) were kindly provided by Professor Rui Zhang (Departments of Immunology, Fourth Military Medical University, Xi'an, China). The sequences of oligonucleotides and plasmids are presented in Table II.

Transfection assay. F4 and F5M2 osteosarcoma cells were plated in 6-well plates at a density of $4 \times 10^{5}$ cells/well, cultured overnight and transfected with $50 \mathrm{nmol} / \mathrm{l}$ oligonucleotides using Lipofectamine ${ }^{\circledR} 2000$ (Invitrogen; Thermo Fisher Scientific, Inc.) for $8 \mathrm{~h}$ at $37^{\circ} \mathrm{C}$. The cells were continuously cultured for $48 \mathrm{~h}$ before the commencement of other experiments.
Lentiviral infection was performed to stably increase or reduce intracellular levels of miR-193b in F5M2 or F4 cells, respectively. Briefly, $2 \times 10^{5}$ cells were plated in a 6 -well plate $24 \mathrm{~h}$ prior to infection. Fresh culture media containing $8 \mu \mathrm{g} / \mathrm{ml}$ polybrene was added to the cells when they reached $60-70 \%$ confluence. Lentivirus (multiplicity of infection, 5 ) was then added to the culture media. After $24 \mathrm{~h}$, viral supernatant was removed and fresh culture medium was added. After incubation for $72 \mathrm{~h}$, the cells were subcultured into $2 \times 100 \mathrm{~mm}$ dishes and $1.0 \mu \mathrm{g} / \mathrm{ml}$ puromycin was added for selection. After antibiotic selection for 2 weeks, the stable clones were obtained.

Cell proliferation assay. $\mathrm{F} 4$ cells transfected with the miR-193b inhibitor or infected with LV-miR-193b inhibitor, and F5M2 cells transfected with miR-193b or infected with LV-193b were maintained in 96-well plates at a density of $2 \times 10^{3}$ cells/well. After $48 \mathrm{~h}$ of culture, cells were treated with $0.5 \%$ MTT solution for $4 \mathrm{~h}$ at $37^{\circ} \mathrm{C}$. After formazan crystals were dissolved in dimethyl sulfoxide, the proliferation rate was measured as the optical density at an absorbance of $570 \mathrm{~nm}$. The experiment was repeated three times with five replicates.

Colony formation assay. A total of 200 cells/well were plated in a 6-well plate and cultured for 2 weeks. Colonies were counted after they were fixed with $100 \%$ methanol for 20 min and stained with $0.1 \%$ crystal violet in $20 \%$ methanol for $30 \mathrm{~min}$ at room temperature. After the cells were washed and dried, visible cell colonies were counted and images were captured. Colony-forming efficiency was calculated using the following equation: Total number of visible cell colonies/200x100. The experiment was repeated three times with five replicates.

Cell cycle analysis. Cell cycle analysis was conducted using a cell cycle detection kit (Beyotime Institution of Biotechnology). Cells were collected and fixed in $75 \%$ ethanol at $4^{\circ} \mathrm{C}$ overnight. After washing with PBS, the cells were treated with $1 \mathrm{mg} / \mathrm{ml}$ RNase at $37^{\circ} \mathrm{C}$ for $40 \mathrm{~min}$. Subsequently, the cells were stained with $50 \mu \mathrm{g} / \mathrm{ml}$ propidium iodide (PI) for $30 \mathrm{~min}$ at $4^{\circ} \mathrm{C}$ in the dark. Cell cycle distribution was analyzed on a COULTER FC500 MCL flow cytometer (Beckman Coulter, Inc.) using MultiCycle for Windows 32-bit software (Beckman Coulter, Inc.). The experiment was repeated three times with five replicates.

Cell apoptosis analysis. Cells $\left(1 \times 10^{3}\right.$ cells $\left./ \mathrm{ml}\right)$ were harvested and washed, then stained with FITC-conjugated Annexin $\mathrm{V}$ (Beyotime Institute of Biotechnology) and PI (Beyotime Biotechnology) at room temperature in the dark for $15 \mathrm{~min}$. Cell apoptosis was measured on a COULTER FC500 MCL flow cytometer (Beckman Coulter, Inc.) using EXPO32 ADC software (Beckman Coulter, Inc.). Apoptotic index was calculated by adding the proportion of Annexin V(+)/PI(-) early apoptotic cells to that of Annexin $\mathrm{V}(+) / \mathrm{PI}(+)$ late apoptotic cells. The experiment was repeated three times with five replicates.

Wound-healing assay. F4 and F5M2 cells at a density of $4 \times 10^{5}$ cells/well were seeded on 6-well plates and cultured in 
Table I. Primers used in the study.

\begin{tabular}{|c|c|}
\hline Gene & Sequence \\
\hline \multicolumn{2}{|c|}{ Primers for construction of KRAS/STMN1 expression vector } \\
\hline KRAS-F & 5'-CAGATCTATGACTGAATATAAACTTGTGG-3' \\
\hline KRAS-R & 5'-GCCTCGAGTTACATAATTACACACTTTGTC-3' \\
\hline STMN1-F & 5'-CAGATCTATGGCTTCTTCTGATATCCAGG-3' \\
\hline STMN1-R & 5'-GCCTCGAGTTAGTCAGCTTCAGTC-3' \\
\hline \multicolumn{2}{|l|}{ Primers for RT-qPCR } \\
\hline Hsa-miR-193b-F & 5'-AACTGGCCCTCAAAGTCCCGCT-3' \\
\hline KRAS-F & 5'-CGGTCATCCAGTGTTGTCAT-3' \\
\hline KRAS-R & 5'-AATGCTCTTGATTTGTCAGCAG-3' \\
\hline STMN1-F & 5'-CCTCTGTTTGGCGCTTTTGTGCG-3' \\
\hline STMN1-R & 5'-GGCACGCTTCTCCAGTTCTTTCACC-3' \\
\hline Actin-F & 5'-TGGCATCCACGAAACTACC-3' \\
\hline Actin-R & 5'-GTGTTGGCGTACAGGTCTT-3' \\
\hline U6-F & 5'-CTCGCTTCGGCAGCACA-3' \\
\hline U6-R & 5'-AACGCTTCACGAATTTGCGT-3' \\
\hline \multicolumn{2}{|c|}{ Primers used in construction of dual-luciferase reporters } \\
\hline (wild type) KRAS-1F & 5'-CGGAATTCGGTGTTGATGATGCCTTC-3' \\
\hline (wild type) KRAS-1R & 5'-GCTGCAGATCATCATCAGGAAGCCC-3' \\
\hline (wild type) KRAS-2F & 5'-CGGAATTCAGGCAGACCCAGTATGAA-3' \\
\hline (wild type) KRAS-2R & 5'-GCTGCAGAATGTCTTGGCACACCACCA-3' \\
\hline (mutant) KRAS-1F & 5'-CGGAATTCGAACTAGCAATGCCTGTG-3' \\
\hline (mutant) KRAS-1R & 5'-GCTGCAGATCATCATCAGGAAGCCC-3' \\
\hline (mutant) KRAS-2F & 5'-GCGAATTCAGAGACCAAGGTTGCAAG-3' \\
\hline (mutant) KRAS-2R & 5'-GCTGCAGAATGTCTTGGCACACCACCA-3' \\
\hline (wild type) STMN1-F & 5'-GCGGAATTCTTGTTCTGAGAACTGACTTTC-3' \\
\hline (wild type) STMN1-R & 5'-GCTGCAGGTCATTTGTGCGTTGGGTAT-3' \\
\hline (mutant) STMN1-F & 5'-GCGGAATTCAATGGCTAGTACTGTATTGG-3' \\
\hline (mutant) STMN1-R & 5'-GCTGCAGGTCATTTGTGCGTTGGGTAT-3' \\
\hline \multicolumn{2}{|c|}{$\begin{array}{l}\text { Primers used in construction of promoter reporters } \mathrm{s}^{\mathrm{a}, \mathrm{b}} \\
\mathrm{t} 1 \text { fragment }\end{array}$} \\
\hline 193b-5'-UTR-1F & 5'-GAGCTCTCGAGTTGGCTTTAGCTTCTGC-3' \\
\hline 193b-5'-UTR-1R & 5'-AGATCTACAGCCTCCAAAAGCCTC-3' \\
\hline \multicolumn{2}{|l|}{ t2 fragment } \\
\hline 193b-5'-UTR-2F & 5'-TTTGAGCTCACGCTTGTCTGGGCTGCGATT-3' \\
\hline 193b-5'-UTR-2R & 5'-CTCGAGGCAGAAGCTAAAGCCAAC-3' \\
\hline \multicolumn{2}{|l|}{ Primers for ChIP assay } \\
\hline c193b-F1 & 5'-TCCGTGCCCCCTGTTTGAA-3' \\
\hline c193b-R1 & 5'-CAGAGAGGGCGAGAGCCTGGAA-3' \\
\hline c193b-F2 & 5'-TGAGTGCTCCCCTTCTTCC-3' \\
\hline c193b-R2 & 5'-AATCGCAGCCCAGACAAGCGT-3' \\
\hline c193b-F3 & 5'-CCTGGGCTTGGAAATTGAC-3' \\
\hline c193b-R3 & 5'-GGTAACTCTCTGGGGACGGT-3' \\
\hline c193b-F4 & 5'-GGCGCGCAGCAAATTTGACT-3' \\
\hline c193b-R4 & 5'-TTGATCCCGGGGTGTCTCTT-3' \\
\hline c193b-F5 & 5'-GGAGGAGAAAGTACATTCCC-3' \\
\hline c193b-R5 & 5'-TGTTGCAATTCCAGGTGGAAGC-3' \\
\hline
\end{tabular}

${ }^{a}$ Wild type $=\mathrm{t} 1+\mathrm{t} 2$ fragments; ${ }^{\mathrm{b}}$ mutant=t 1 fragment. F, forward; miR, microRNA; R, reverse; RT-qPCR, reverse transcription-quantitative PCR; STMN1, stathmin 1; UTR, untranslated region.

RPMI medium supplemented with $10 \%$ fetal bovine serum. When the cells reached $100 \%$ confluence after $24 \mathrm{~h}$ of culture, a wound-healing assay was performed. A $20 \mu 1$ pipette tip was used to make three parallel wounds. After the cells were cultured in serum-free medium for 48 or $96 \mathrm{~h}$, images were captured under a light microscope and analyzed with ImageJ 
Table II. Oligonucleotides sequences used for transfection.

\begin{tabular}{ll}
\hline Oligonucleotide & \multicolumn{1}{c}{ Sequence } \\
\hline miR-193b mimic & F: 5'-AACUGGCCCUCAAAGUCCCGCU-3' \\
NC & R:5'-CGGGACUUUGAGGGCCAGUUUU-3' \\
& F: 5'-UUCUCCGAACGUGUCACGUTT-3' \\
miR-193b inhibitor & R: 5'-ACGUGACACGUUCGGAGAATT-3' \\
Inhibitor NC & 5'-AGCGGGACUUUGAGGGCCAGUU-3' \\
siNC & 5'-CAGUACUUUUGUGUAGUACAA-3' \\
siKRAS & F: 5'-UUCUCCGAACGUGUCACGUTT-3' \\
siSTMN1 & R: 5'-ACGUGACACGUUCGGAGAATT-3' \\
siMYC & F: 5'-GGGCUUUCUUUGUGUAUUUTT-3' \\
& R: 5'-AAAUACACAAAGAAAGCCCTT-3' \\
& F: 5'-GAUGUUUAUUUGCAAACAACC-3' \\
& R: 5'-UUGUUUGCAAAUAAACAUCUG-3' \\
& F: 5'-AACGUUAGCUUCACCAACAUU-3' \\
& R: 5'-UGUUGGUGAAGCUAACGUUUU-3'
\end{tabular}

F, forward; miR, microRNA; NC, negative control; R, reverse; si, small interfering RNA; STMN1, stathmin 1.

(version 1.8.0; National Institutes of Health). The experiment was repeated three times with three replicates.

Transwell cell invasion assay. The upper chamber of Transwell culture plates ( $8 \mu \mathrm{m}$; Corning, Inc.) was coated with $0.4 \mathrm{mg} / \mathrm{ml}$ Matrigel (BD Biosciences) and incubated for $24 \mathrm{~h}$ at $4^{\circ} \mathrm{C}$. Cells were seeded into the upper chamber of Transwell culture plates at a density of $2 \times 10^{5}$ cells/well. The lower chamber was filled with RPMI supplemented with $10 \%$ fetal bovine serum. After $36 \mathrm{~h}$, cells on the upper membrane that did not migrate were wiped with a cotton swab, and the membrane in the lower chamber was fixed with absolute ethyl alcohol and stained with $0.1 \%$ crystal violet for $30 \mathrm{~min}$ at room temperature. The number of migrated cells was then counted under a light microscope in at least five fields. The experiment was repeated three times with three replicates.

Reverse transcription-quantitative PCR (RT-qPCR). Total RNA (containing small RNAs) was extracted using TRIzol ${ }^{\circledR}$ reagent (Invitrogen; Thermo Fisher Scientific, Inc.) and reversed transcribed into cDNA. For miR-193b, RT was conducted at $37^{\circ} \mathrm{C}$ for $1 \mathrm{~h}$, followed by $85^{\circ} \mathrm{C}$ for 5 sec using the PrimeScript miRNA qPCR Starter kit ver. 2.0 (Takara Biotechnology Co., Ltd.). The forward primer for miR-193b was 5'-AACTGGCCCTCAAAGTCCCGCT-3' as described in Table I, while the reverse primer was a universal primer from the PrimeScript ${ }^{\mathrm{TM}}$ miRNA qPCR Starter kit. The expression levels of miR-193b were quantified by qPCR in a real-time PCR detector (Bio-Rad Laboratories, Inc.) using the PrimeScript ${ }^{\mathrm{TM}}$ miRNA qPCR Starter kit ver. 2.0. For the quantitative detection of KRAS, STMN1 and MYC mRNA, RT was conducted at $37^{\circ} \mathrm{C}$ for $15 \mathrm{~min}$, followed by $85^{\circ} \mathrm{C}$ for $5 \mathrm{sec}$ with a PrimeScript RT reagent kit (Bio-Rad Laboratories, Inc.). Subsequently, qPCR was conducted using the SYBR Green PCR Master Mix kit (Applied Biosystems; Thermo Fisher Scientific, Inc.). Two-step PCR amplification was conducted at $95^{\circ} \mathrm{C}$ for $180 \mathrm{sec}$, followed by 40 cycles at $95^{\circ} \mathrm{C}$ for $10 \mathrm{sec}$ and $57^{\circ} \mathrm{C}$ for $30 \mathrm{sec}$. Final extension was performed at $72^{\circ} \mathrm{C}$ for $5 \mathrm{~min}$. U6 and GAPDH served as internal controls for miRNA and mRNA expression levels, respectively. Relative expression levels were calculated using the $2^{-\Delta \Delta \mathrm{Cq}}$ method (20). All primers used for RT-qPCR analysis are listed in Table I. The experiment was carried out in triplicate and repeated three times.

Western blot analysis. After cells were lysed with RIPA lysis buffer (Beyotime Institute of Biotechnology), the supernatant was harvested by centrifugation at $14,000 \mathrm{x}$ g for $30 \mathrm{~min}$ at $4^{\circ} \mathrm{C}$. Protein quantification was performed using the BCA method and $30 \mu \mathrm{g}$ protein was loaded on SDS-polyacrylamide gels for SDS-PAGE. Proteins were resolved on a $12 \%$ precast gradient gel and were then transferred to a nitrocellulose membrane. The membranes were blocked with $5 \%$ (w/v) non-fat dry milk in TBS-0.05\% Tween (Beyotime Institute of Biotechnology) for $1 \mathrm{~h}$ at room temperature and were then incubated with primary antibodies overnight at $4^{\circ} \mathrm{C}$, followed by incubation with a secondary antibody (1:3,000; cat. nos. 7076 or 5127 ; Cell Signaling Technology, Inc.) for $1 \mathrm{~h}$ at room temperature. To visualize protein expression, enhanced chemiluminescence (ECL) was performed with the Pierce ${ }^{\mathrm{TM}}$ ECL Western Blotting Substrate kit (Thermo Fisher Scientific, Inc.). The gray value of the target band was analyzed using Image Lab (Version 3.1; Bio-Rad Laboratories, Inc.). Antibodies against KRAS (1:250; F234; cat. no. sc-30; Santa Cruz Biotechnology, Inc.), STMN1 (1:1,000; cat. no. ab131481; Abcam), MYC (1:1,000; cat. no. ab32072; Abcam), and actin (1:2,000; cat. no. A5441; Sigma-Aldrich; Merck KGaA) were used. The experiment was performed in triplicate.

Dual-luciferase reporter assay. Human KRAS-3'-untranslated region (3'-UTR) and STMN1-3'-UTR reporter constructs containing the putative binding site of miR-193b, and their 
identical sequences with a mutation in the seed sequence, were amplified by PCR using the primers listed in Table I as described previously (21), and inserted between the EcoRI and PstI restriction sites of the PGL3-basic reporter vector (Promega Corporation). There are two binding sites for miR-193b on KRAS 3'UTR. Thus, to clarify whether the two binding sites are both effective, two reporter vectors for KRAS with the corresponding binding sites were constructed. 293A cells at a density of $2 \times 10^{5}$ cells/well were co-transfected with $50 \mathrm{nM}$ miR-193b mimic or NC mimic and $1 \mathrm{mg}$ reporter vectors containing wild-type or mutant 3'-UTR of KRAS or STMN1 according to the aforementioned transfection protocol. At $6 \mathrm{~h}$ post-transfection, the medium was replaced with complete medium and at $48 \mathrm{~h}$ post-transfection, cells were lysed and luciferase activity was detected using the Dual-Luciferase Assay kit (Promega Corporation). The primers used to amplify the wild-type and mutant 3'-UTRs by PCR are listed in Table I. Each sample was tested in triplicate, and the experiment was repeated three times.

Promoter reporter assay. A $1.0 \mathrm{~kb}$ wild-type promoter fragment of miR-193b with a putative MYC binding site, or a truncated promoter fragment of miR-193b without a MYC binding site (synthesized by Sangon Biotech Co., Ltd.) was PCR-amplified and then cloned into the SacI and BglII sites of a pGL3-Basic vector (Promega Corporation). 293A cells were seeded in 24-well at a density of $2 \times 10^{5}$ cells/well and were cultured until confluence reached $80 \%$. Subsequently, the cells were co-transfected with $400 \mathrm{ng}$ wild-type or truncated reporter vector together with $400 \mathrm{ng}$ pBabe or pBabe-MYC plasmid. The pTK-luc (Renilla) luciferase vector (20 ng; Thermo Fisher Scientific, Inc.) was transfected into 293A cells together with the reporter vectors and pBabe plasmids for normalization. The luciferase activity was measured $48 \mathrm{~h}$ post-transfection using the Dual-Luciferase Reporter Assay system (Promega Corporation) according to the manufacturer's instructions.

Chromatin immunoprecipitation (ChIP)-qPCR. Cells $\left(3 \times 10^{7}\right)$ were fixed with $1.5 \%$ formaldehyde for $10 \mathrm{~min}$ at room temperature and quenched with glycine. After cell lysis with RIPA buffer (Beyotime Institute of Biotechnology), chromatin was fragmented into 100-500 bp fragments using an ultrasonic cell breaker (DH92-IIN; Lawson Scientific Ltd.) with a cycle of $1 \mathrm{sec}$ on/ $1 \mathrm{sec}$ off for $8 \mathrm{~min}$ (frequency, $20 \mathrm{kHz}$ ). Protein-DNA complexes were immunoprecipitated with MYC antibody (cat. no. ab32072; Abcam) or anti-IgG antibody (cat.no. ab171870; Abcam) conjugated with Dynabeads Protein G (cat. no. 10007D; Invitrogen; Thermo Fisher Scientific, Inc.) as described previously (22). After being eluted from the beads using ChIP Elution Buffer (cat. no. 714231S; Cell Signaling Technology, Inc.) for $30 \mathrm{~min}$ at $65^{\circ} \mathrm{C}$, the antibody-protein-DNA complex was reverse cross-linked by incubation at $65^{\circ} \mathrm{C}$ with $200 \mathrm{mM} \mathrm{NaCl}$. Six primers were designed according to the predicted binding sites on miR-193b (sequences are listed in Table I), and quantification of immunoprecipitated DNA was performed by RT-qPCR. Each sample was tested in triplicate, and the experiment was repeated three times.

In vivo tumorigenicity and metastasis analysis. Female $\mathrm{BALB} / \mathrm{c}$ nude-mice (age, 6 weeks; weight, $20 \mathrm{~g} ; \mathrm{n}=5 \mathrm{mice} / \mathrm{group}$ ) were obtained and maintained at the Qualified Animal Care Facility, The Fourth Military Medical University. During the study, animal welfare was seriously taken into consideration. All animal handling and experimental procedures were approved by the Animal Ethics Committees of The Fourth Military Medical University and were in accordance with the guidelines of the China Council of Animal Care (Regulation for the Administration of Affairs Concerning Experimental Animal approved by Decree No. 2 of the State Science and Technology Commission on November 14th, 1988). All mice were housed in polycarbonate cages, provided with free access to food and water, and maintained under a $12 \mathrm{~h}$ light-dark cycle at $22 \pm 2^{\circ} \mathrm{C}$ with $60 \pm 5 \%$ humidity. Humane endpoints and clinical signs used in all experiments to determine when to euthanize the mice include: Tumors $2.0 \mathrm{~cm}$ diameter, weight loss $>20 \%$, tumor ulceration, abnormal posture, quick weight loss, loss of appetite for $>3$ days, and too weak to access water and food. However, no experimental mice met these criteria and were euthanized before the end of our experiments. On the indicated time of sacrifice, mice were anesthetized with isoflurane (4\%) for $\sim 1 \mathrm{~min}$. Animals with no heartbeat for $30 \mathrm{sec}$ accompanied by no response to the toe pinch reflex were considered to be dead. Subsequently, cervical dislocation was performed to verify euthanasia. In the tumor formation in vivo experiment, the maximum tumor burden was $<4 \%$ animal weight.

For the tumorigenicity study, female BALB/c nude-mice (age, 6 weeks; weight, $20 \mathrm{~g}$; $\mathrm{n}=5$ mice/group) were anesthetized with isoflurane after initial induction in a chamber provided with oxygen $(2 \mathrm{l} / \mathrm{min})$ containing $2 \%$ isoflurane, in order to minimize suffering and pain during cell injection. F5M2 cells stably transfected with LV-miR-193b or LV-NC were subcutaneously injected into the dorsal flank of the mice. When tumors were palpable on day 4 , the tumor diameter was measured and documented every 5 days until day 35. Tumor volume was calculated as follow: Volume $=$ width $^{2} \mathrm{x}$ length x0.5 (23). The animals were sacrificed on day 35 , and the tumors were collected, weighed and photographed. For the metastasis study, F5M2 cells transfected with LV-NC or LV-miR-193b were injected into the tail vein of mice. A total of 6 weeks after tumor cell implantation, mice were euthanized, and the lungs of the experimental animals were collected, photographed and weighed. Subsequently, the number of tumor nodules formed on the lung surfaces was counted. Metastatic lungs were fixed in $4 \%$ paraformaldehyde (Beyotime Institute of Biotechnology) for $24 \mathrm{~h}$ at room temperature, and then embedded with paraffin. Serial sections of lungs were obtained and stained with hematoxylin and eosin to confirm the pulmonary metastatic lesions under a light microscope.

Immunofluorescence staining. Paraffin-embedded sections were dewaxed and endogenous peroxidase was removed using $3 \% \mathrm{H}_{2} \mathrm{O}_{2}$ for $10 \mathrm{~min}$ at room temperature. Heat-mediated antigen retrieval was performed by immersing the sections in citrate buffer (Abcam) and boiling them in a microwave oven for $10 \mathrm{~min}$. Subsequently, the sections were cooled, washed and blocked in PBS containing 2\% goat serum (Thermo Fisher Scientific, Inc.) and $0.1 \%$ Triton X-100 for $60 \mathrm{~min}$ at room temperature. Subsequently, the sections were incubated with 
A

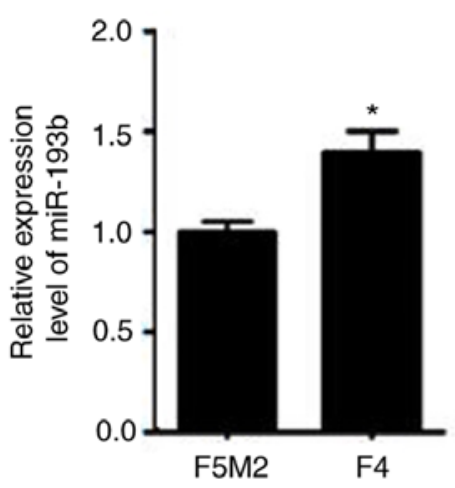

B

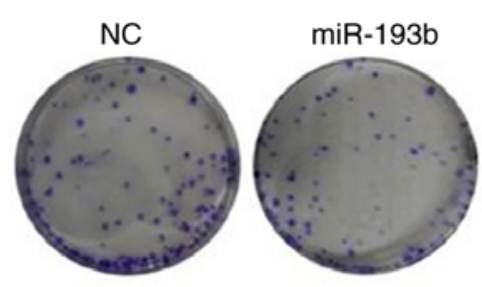

F4

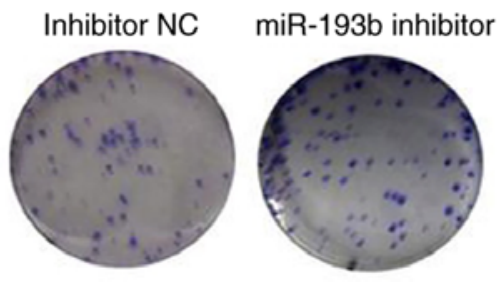

F4

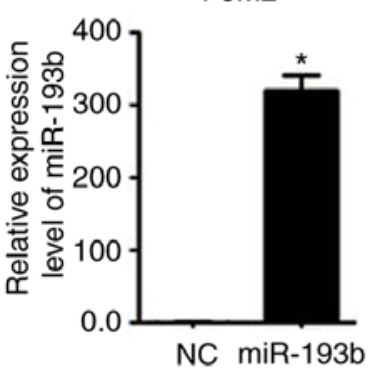

F5M2

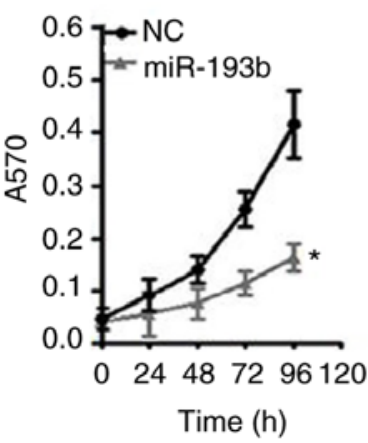

E

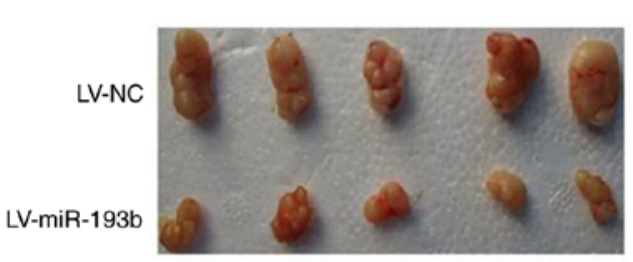

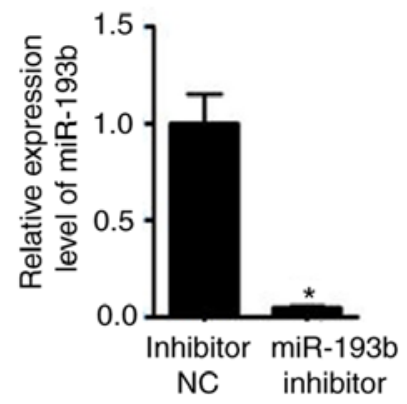

F4

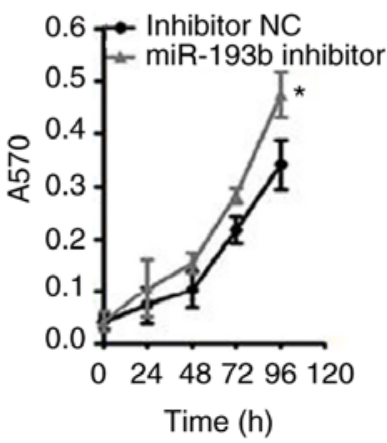

F5M2

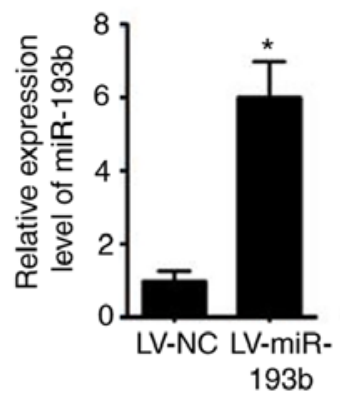

F
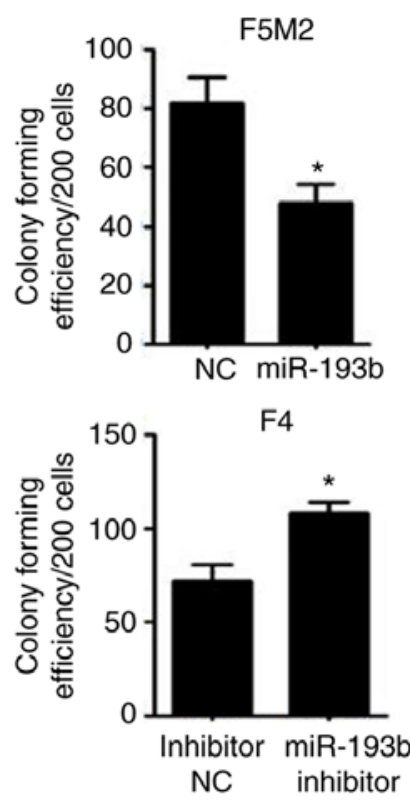

F4

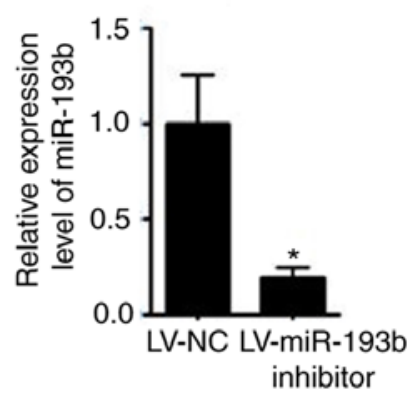

F4
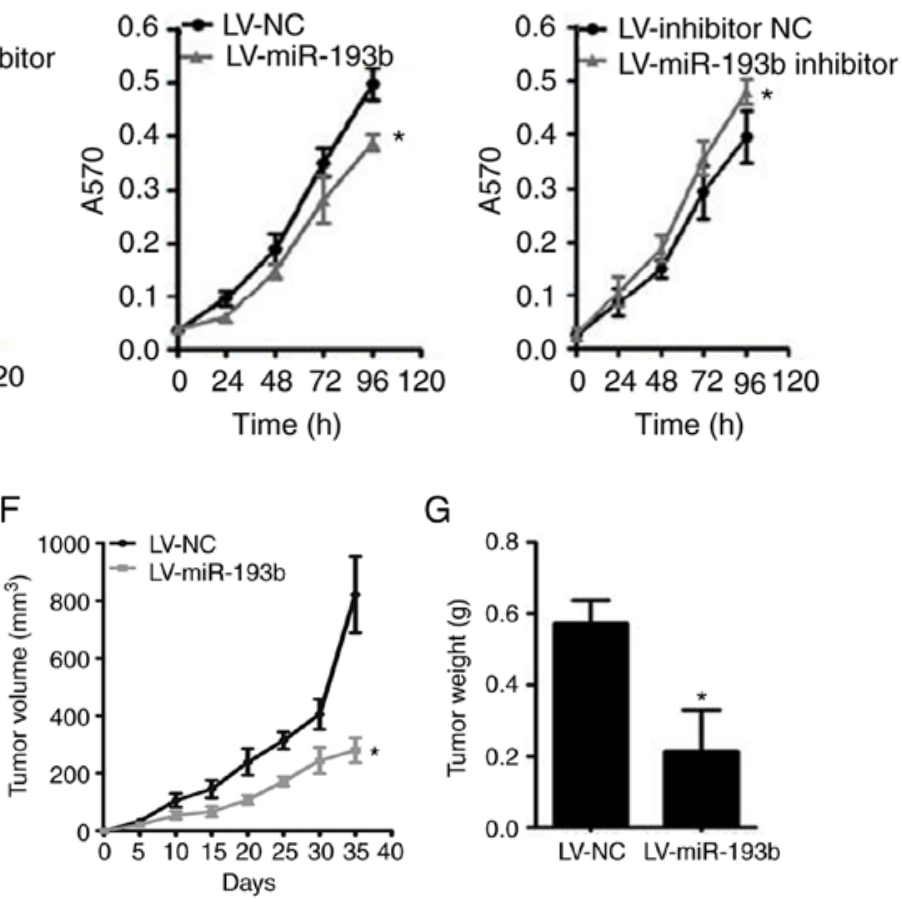

G

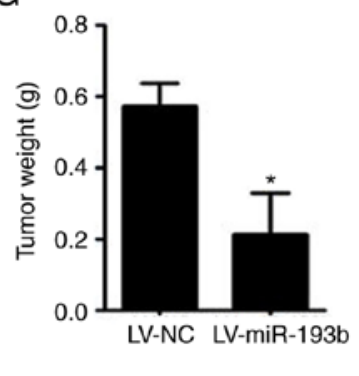

Figure 1. miR-193b suppresses proliferation and colony formation of osteosarcoma cells, and inhibits tumor growth in a xenograft model. (A) miR-193b expression was lower in F5M2 cells compared with F4 cells. (B) Overexpression of miR-193b reduced colony formation of F5M2 cells, whereas downregulation of miR-193b enhanced colony formation of F4 cells. (C) Transient and stable transfection efficiencies of miR-193b mimic or inhibitor were quantified. (D) Transient or stable transfection of miR-193b mimic in F5M2 cells inhibited proliferation, whereas transfection of miR-193b inhibitor in F4 cells enhanced cell proliferation. (E) Representative images illustrating tumor formation in a nude mouse xenograft model on day 35. (F) Tumor volume and (G) tumor weight were lower in mice injected with miR-193b-upregulated F5M2 cells. Data are presented as the mean \pm SD of at least three independent experiments. "P<0.05. LV, lentivirus; miR-193b, microRNA-193b; NC, negative control. 
primary antibodies against KRAS (1:200; cat. no. sc-30; Santa Cruz Biotechnology, Inc.), STMN1 (1:200; cat. no. ab221017; Abcam) or MYC (1:200; cat. no. ab39688; Abcam) overnight at $4^{\circ} \mathrm{C}$. The sections were then washed and incubated with Alexa Fluor ${ }^{\circledR}$ 488-conjugated goat anti-mouse IgG antibody (1:500; cat. no. ab150113; Abcam) for $1 \mathrm{~h}$ at room temperature. After washing, DAPI nuclear staining was performed. The stained sections were observed under an Olympus IX53 inverted fluorescence microscope (Olympus Corporation). The experiment was performed in triplicate.

Bioinformatics analysis. The target genes of miR-193b were predicted by computer-aided algorithms using TargetScan (http://www.targetscan.org/, release 7.1: June 2016) and Microcosm Targets (http://www.mirbase.org/, release 21: 2014). The transcription factors that could bind to the promoter region of miR-193b were predicted using Genome Browser from National Center for Biotechnology Information (http://www.ncbi.nlm.nih.gov/), University of California Santa Cruz (http://www.genome.ucsc.edu/), and Ensembl (http://www.ensembl.org/index.html).

Statistical analysis. Each experiment was repeated at least three times. The data are presented as the mean \pm standard deviation. SPSS V18.0 software (SPSS, Inc.) was used for statistical analysis. The normality of data from various groups was tested using the Kolmogorov-Smirnov test and homogeneity was tested using the Levene test. The statistical significance of differences between two groups was evaluated using unpaired Student's t-test (two-tailed). For comparisons of datasets containing multiple groups, one-way ANOVA and post-hoc Tukey test was performed. $\mathrm{P}<0.05$ was considered to indicate a statistically significant difference.

\section{Results}

miR-193b inhibits osteosarcoma growth in vitro and in vivo. When the expression of miR-193b was compared between the two cell lines, F5M2 cells, which have a relatively high metastatic potential, exhibited lower miR-193b expression than F4 cells, which have lower metastatic potential (Fig. 1A).

Subsequently, the expression of miR-193b was transiently or stably altered (Fig. 1C), and the effects of miR-193b on proliferation and colony-forming capacities were investigated. The results revealed that the miR-193b mimic reduced the proliferation and colony-forming capacity of F5M2 cells, whereas the miR-193b inhibitor enhanced these abilities in F4 cells (Fig. 1B and D). Similar results were found in F5M2 and F4 cells with stably altered miR-193b expression (Fig. 1B and D).

As shown in Fig. 1E-G, the xenograft tumor formation assay revealed that the tumor volume and weight were significantly higher in the group injected with wild-type F5M2 cells compared with those injected with F5M2 cells with stably upregulated miR-193b. Detailed information about animal weight, tumor dimension and tumor weight for each experimental animal is provided in Table SI.

miR-193b induces cell cycle arrest and apoptosis. Increasing the expression levels of miR-193b in F5M2 cells induced cell cycle arrest in the $\mathrm{G}_{1}$ phase (Fig. 2A) and significantly increased the percentage of apoptotic cells (Fig. 2C). After knockdown of miR-193b in F4 cells, the percentage of cells in $G_{1}$ phase decreased (Fig. 2B), whereas the proportion of cells in $\mathrm{S}$ and $\mathrm{G}_{2}$ phases was increased. The proportion of apoptotic cells decreased in miR-193b-inhibitor-transfected F4 cells (Fig. 2D).

miR-193b suppresses the migratory and invasive ability of osteosarcoma cells. As shown in Fig. 3A and B, wound-healing assay and Transwell cell invasion assay revealed that transient upregulation of miR-193b reduced the migration and invasion abilities of F5M2 cells, whereas downregulation of miR-193b in F4 cells exerted opposite effects. In addition, when a Transwell cell invasion assay was performed after miR-193b was stably altered, results were consistent with those aforementioned.

The in vivo study revealed that mice injected with miR-193b-upregulated F5M2 cells had significantly lower lung weights and fewer pulmonary metastatic nodules compared with mice injected with wild-type F5M2 cells (Fig. 3C-E).

KRAS and STMN1 are targets of $m i R-193 \mathrm{~b}$. The target molecules of miR-193b were predicted using numerous miRNA target gene prediction websites. KRAS and STMN1 were selected from the predicted targets (Fig. 4A), since they have been associated with the malignant behavior of tumors $(16,17)$. The expression levels of KRAS and STMN1 were higher in F5M2 cells than in F4 cells (Fig. 4B), showing an inverse trend compared with miR-193b. The prediction was further validated using a luciferase reporter assay, which revealed that miR-193b markedly reduced the luciferase activities of the reporter vectors containing wild-type constructs of KRAS and STMN1, but not vectors containing mutant constructs (Fig. 4C). In addition, upregulation of miR-193b in F5M2 cells induced a decrease in KRAS and STMN1 expression (Fig. 4D), whereas the downregulation of miR-193b in F4 cells exerted opposite effects (Fig. 4E). Immunofluorescence staining for KRAS and STMN1 in xenograft tumor sections also revealed that upregulation of miR-193b in osteosarcoma cells reduced the expression of KRAS and STMN1 in vivo (Fig. 4F).

miR-193b inhibits proliferation via KRAS and suppresses migration and invasion via STMN1. Expression levels of KRAS and STMN1 were deliberately enhanced or reduced using pMSCV-KRAS/pMSCV-STMN1 or KRAS siRNA (siKRAS)/STMN1 siRNA (siSTMN1). Transfection efficiency was validated using western blotting and RT-qPCR (Fig. 5A and B). F5M2 cells were transfected with miR-193b mimic plus empty pMSCV or miR-193b mimic plus pMSCV-KRAS. F4 cells were transfected with miR-193b inhibitor plus siNC or miR-193b inhibitor plus siKRAS. The cells were then subjected to cell cycle analysis. As shown in Fig. 5C, pMSCV-KRAS reversed the cell cycle arrest induced by miR-193b in F5M2 cells. Similarly siKRAS abolished the miR-193b inhibitor-induced increase in the percentage of cells in $G_{2}$ phase in F4 cells (Fig. 5C). To study the involvement of STMN1 in miR-193b-mediated regulation of invasion and migration, F5M2 cells were transfected with miR-193b mimic plus empty pMSCV or miR-193b mimic plus pMSCV-STMN1, and F4 cells were transfected with miR-193b inhibitor plus 
A
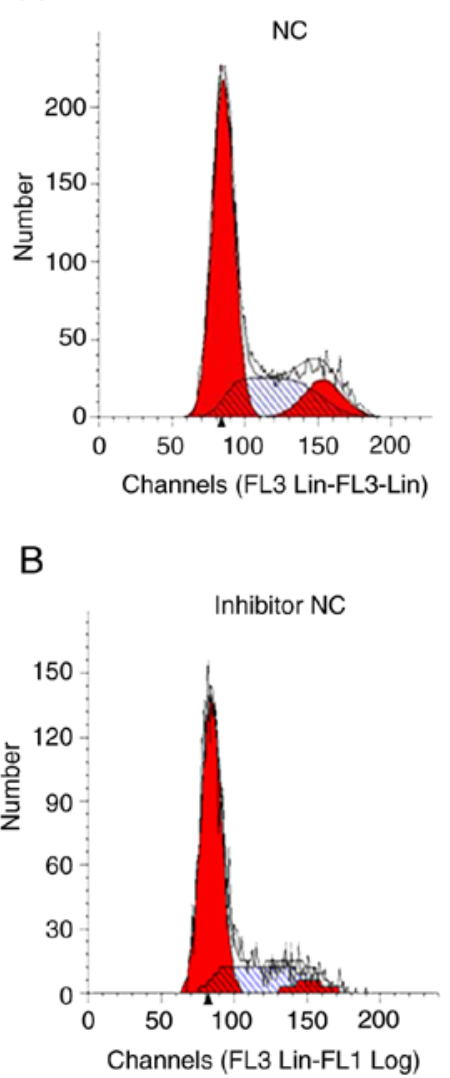

C

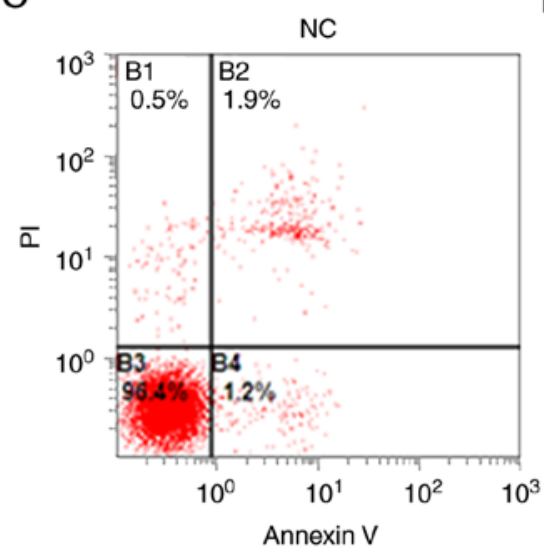

D

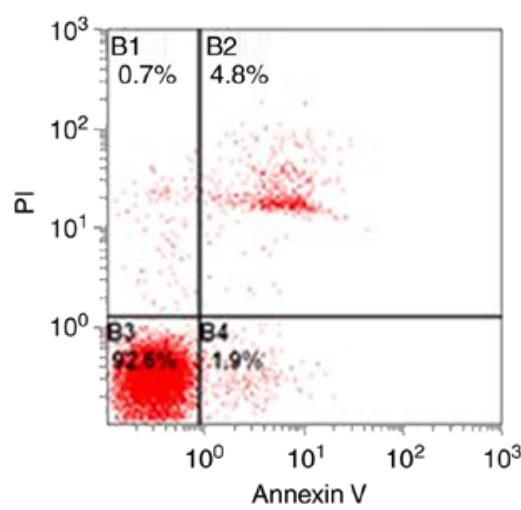

F5M2
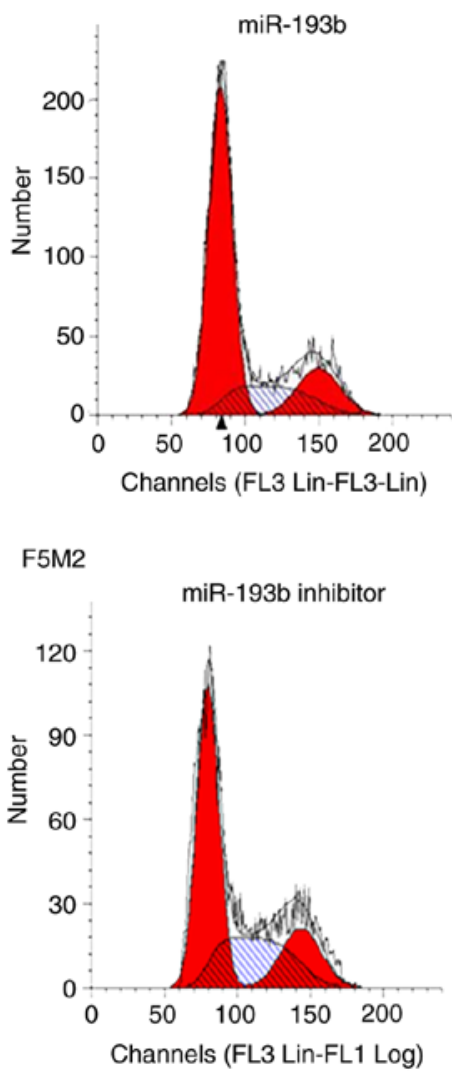

F5M2

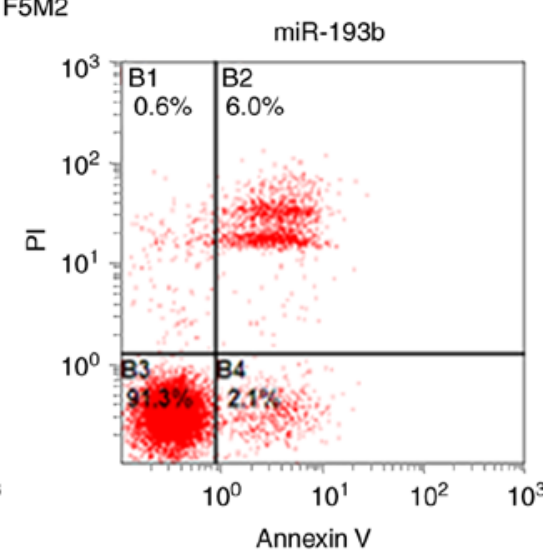

F4

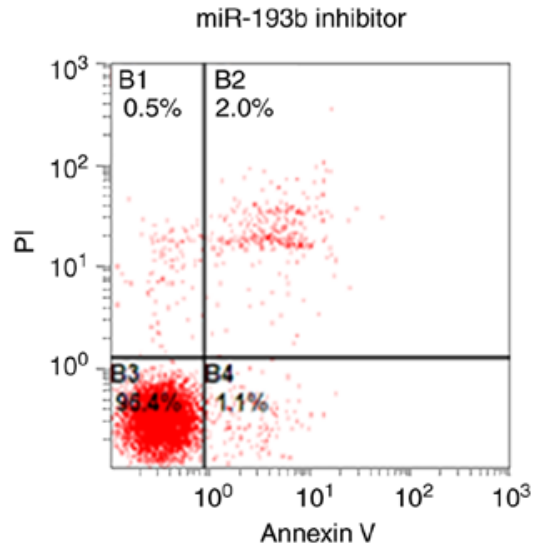

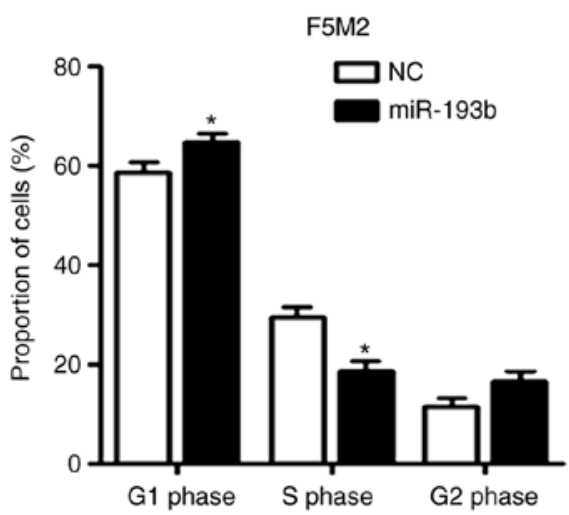

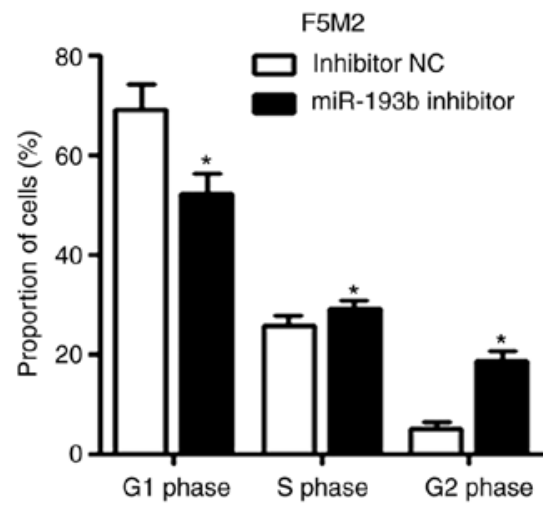

F4

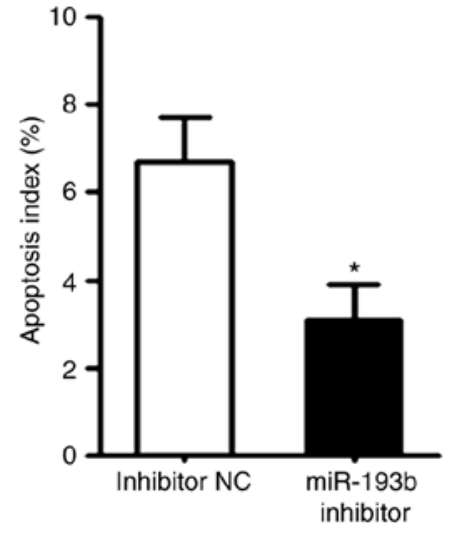

Figure 2. miR-193b inhibits cell cycle progression and induces apoptosis. (A) Transfection of F5M2 cells with miR-193b mimic induced cell cycle arrest in $\mathrm{G}_{1}$ phase, (B) whereas transfection of $\mathrm{F} 4$ cells with miR-193b inhibitor resulted in an increased percentage of cells in $S$ and $\mathrm{G}_{2}$ phases. (C) Upregulation of miR-193b induced apoptosis of F5M2 cells, (D) whereas downregulation of miR-193b in F4 cells inhibited apoptosis. Data are presented as the mean \pm SD of at least three independent experiments. "P<0.05. miR-193b, microRNA-193b; NC, negative control; PI, propidium iodide. 
A

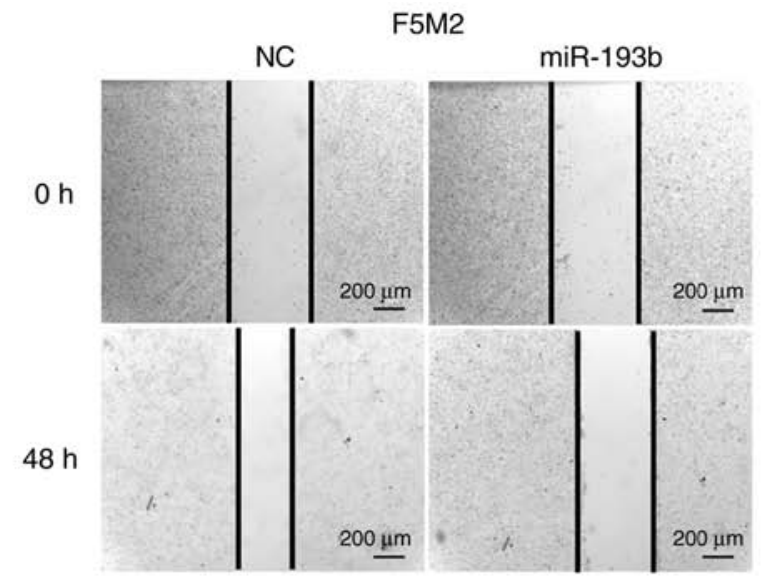

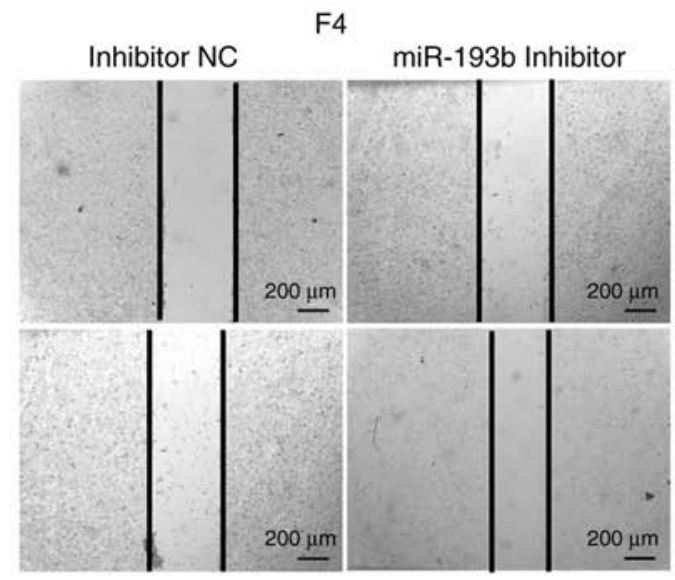

B
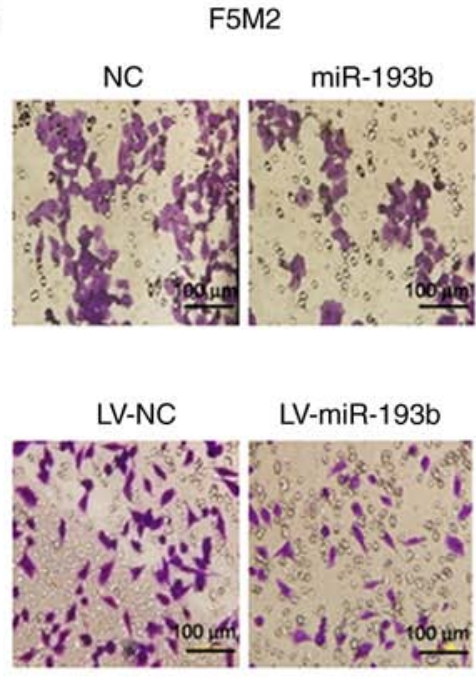
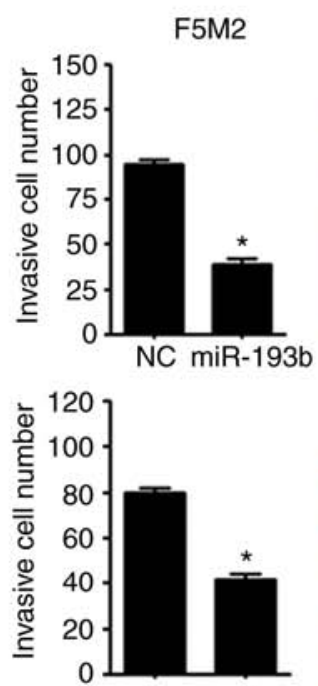
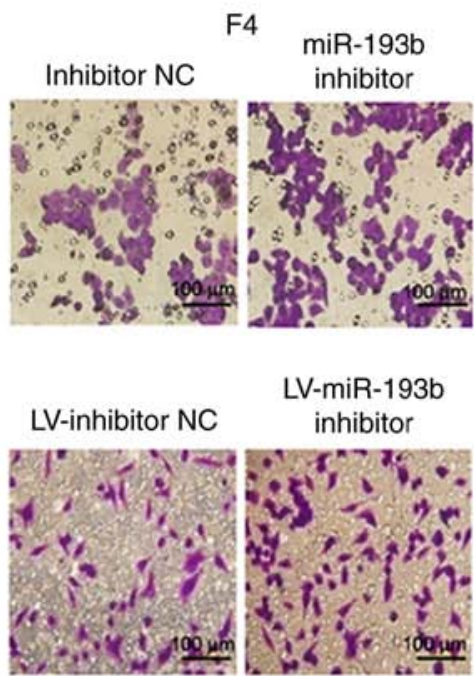

C
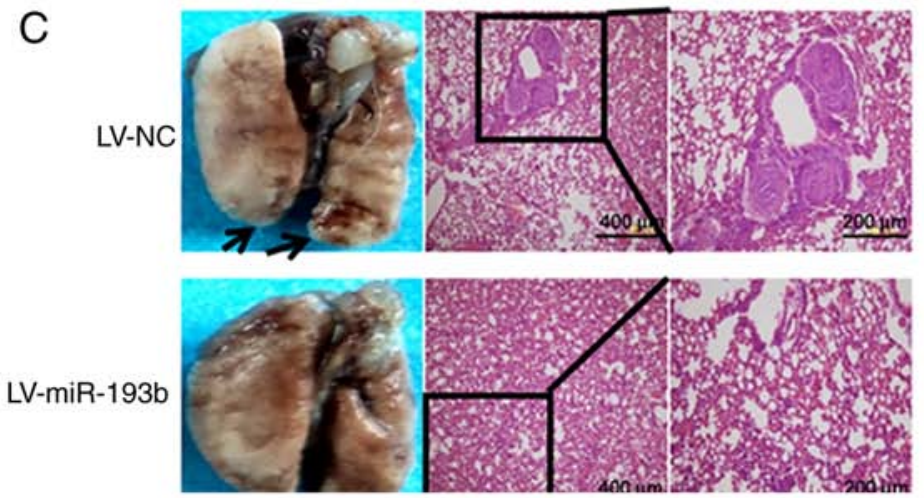
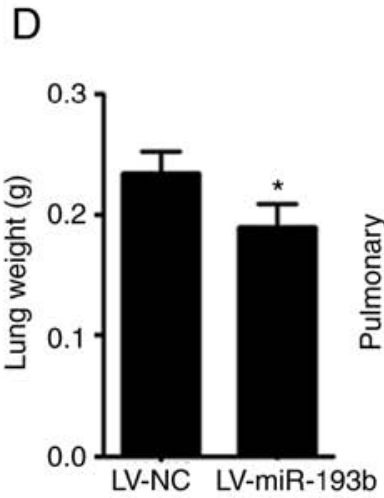

F4
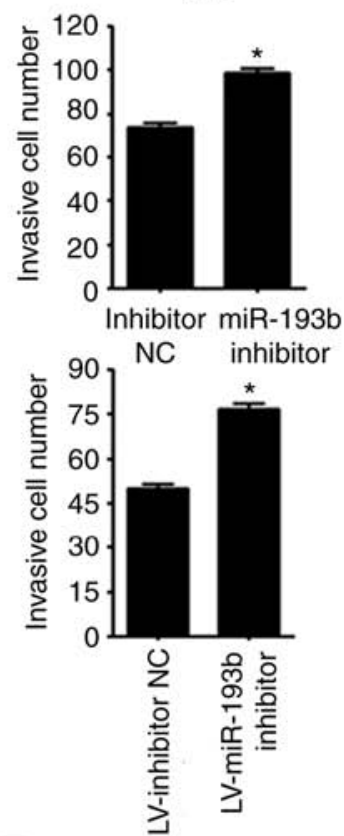

$E$

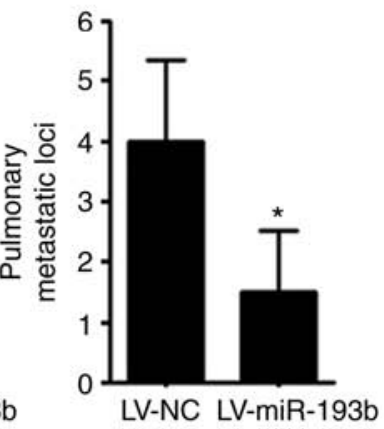

Figure 3. miR-193b inhibits migration and invasion of osteosarcoma cells and reduces the formation of lung metastasis. (A) Wound-healing assay revealed that upregulation of miR-193b inhibited cell migration in F5M2 cells, whereas downregulation of miR-193b induced the opposite result in F4 cells (magnification x20). (B) Transwell cell invasion assay revealed that transient or stable upregulation of miR-193b inhibited invasion of F5M2 cells, whereas downregulation of miR-193b enhanced invasion of F4 cells (magnification x40). (C) Representative images of the lungs and their corresponding H\&E staining pictures (magnification $\mathrm{x} 10$ and $\mathrm{x} 20$ ). Metastatic nodules in the lung were indicated by an arrow. (D) Lung weight and (E) number of microscopic pulmonary metastatic nodules were lower in mice injected with miR-193b-upregulated F5M2 cells. Data are presented as the mean \pm SD of at least three independent experiments. ${ }^{*} \mathrm{P}<0.05$. LV, lentivirus; miR-193b, microRNA-193b; NC, negative control.

siNC or miR-193b inhibitor plus siKRAS. Subsequently, Transwell cell invasion assay and wound-healing assays were performed. As can be seen from the results of Transwell cell invasion assay, co-transfection of F5M2 cells with the miR-193b mimic and pMSCV-STMN1 reversed miR-193b-induced inhibition of invasion, whereas co-transfection of F4 
A

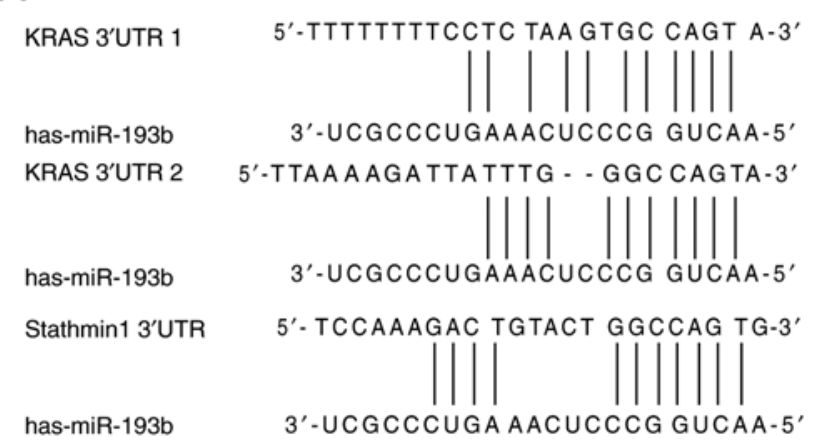

C
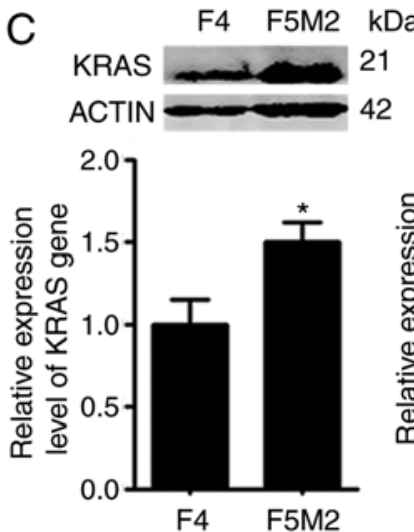

F4 F5M2 kDa
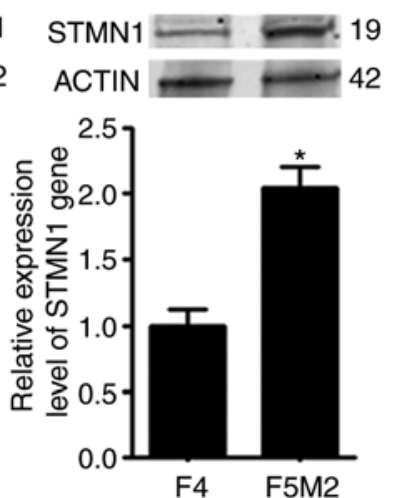

B

PGL3-KRAS 3'UTR1

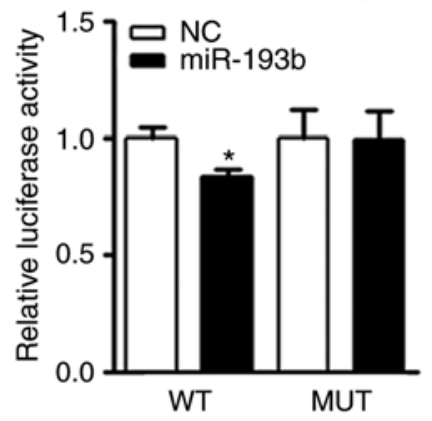

PGL3-KRAS 3'UTR2

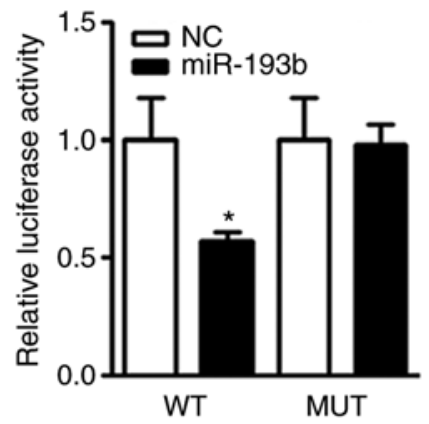

PGL3-STMN1 3'UTR

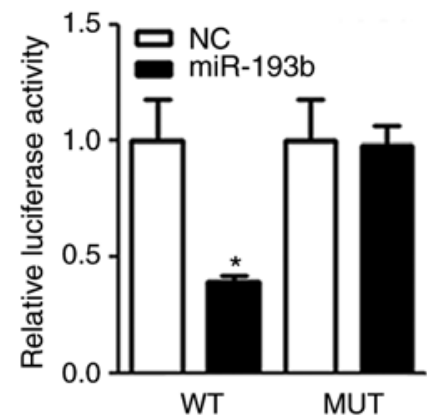

D
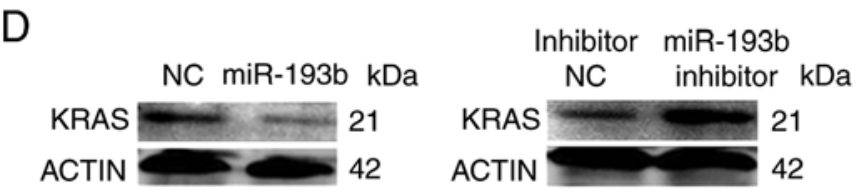

E

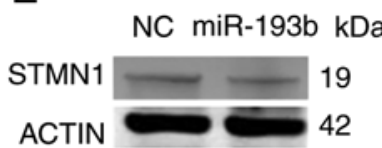

Inhibitor miR-193b $\mathrm{NC}$ inhibitor $\mathrm{kDa}$

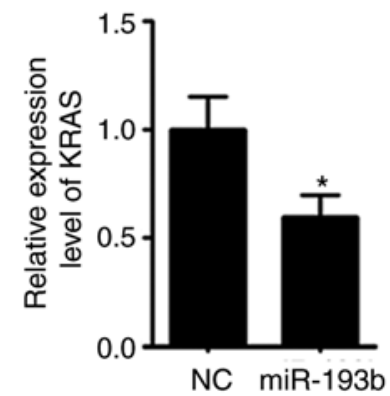

NC miR-193b

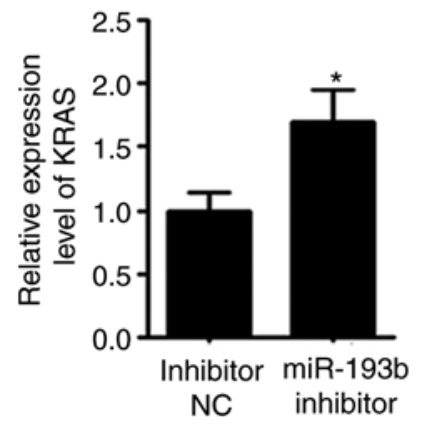

NC inhibitor

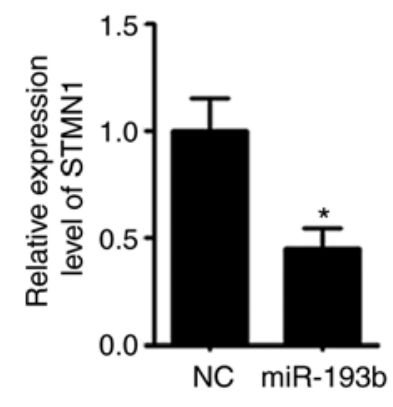

DAPI

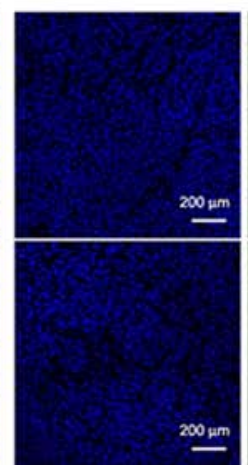

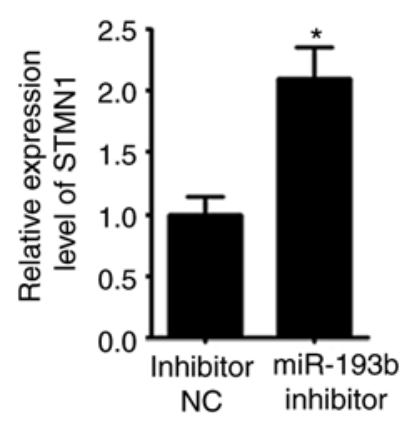

STMN

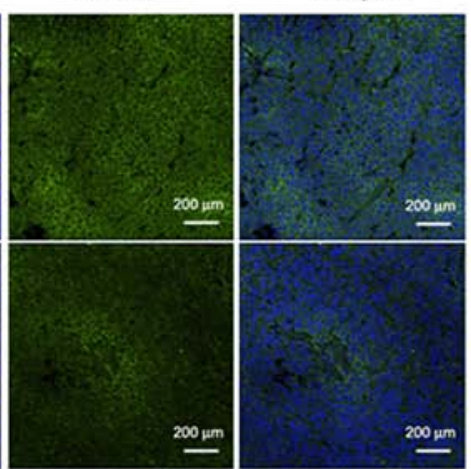

Figure 4. miR-193b suppresses KRAS and STMN1 expression through their 3'-UTRs. (A) Schematic diagram of KRAS and STMN1 3'-UTRs with the locations of predicted conserved miRNA-targeting sequences highlighted. (B) Expression levels of KRAS and STMN1 were higher in F5M2 cells compared with F4 cells. (C) Luciferase reporter assay revealed that miR-193b suppressed luciferase activities of all WT constructs. (D and E) miR-193b negatively regulated the expression of KRAS and STMN1. (F) Immunofluorescence staining revealed that tumor tissue from nude mice injected with miR-193b-upregulated F5M2 cells exhibited lower expression levels of KRAS and STMN1 compared with the control group (magnification x20). Data are presented as the mean \pm SD of at least three independent experiments. "P<0.05. LV, lentivirus; miR-193b, microRNA-193b; MUT, mutant; NC, negative control; STMN1, stathmin 1; UTR, untranslated region; WT, wild-type. 
A

A NC SiKRAS kDa
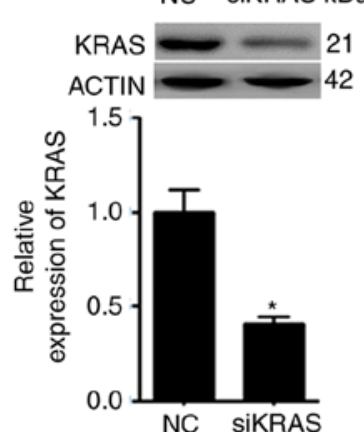

C

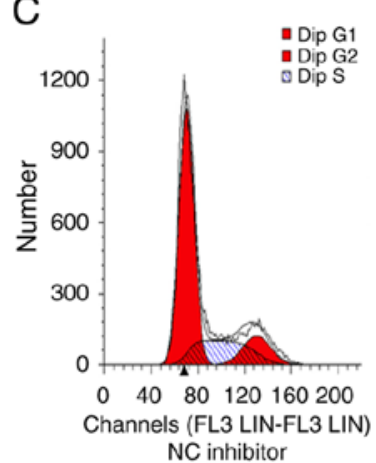

NC inhibitor

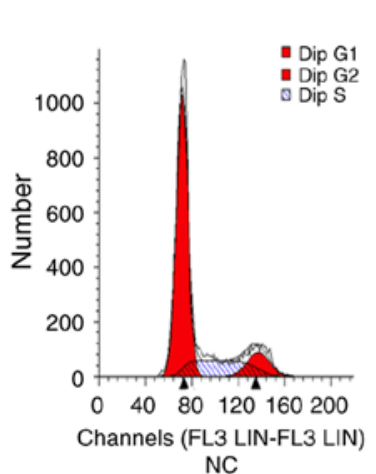

D

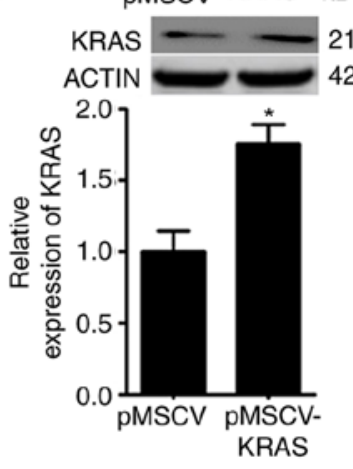

pMSCV-
pMSCV KRAS kDa

B
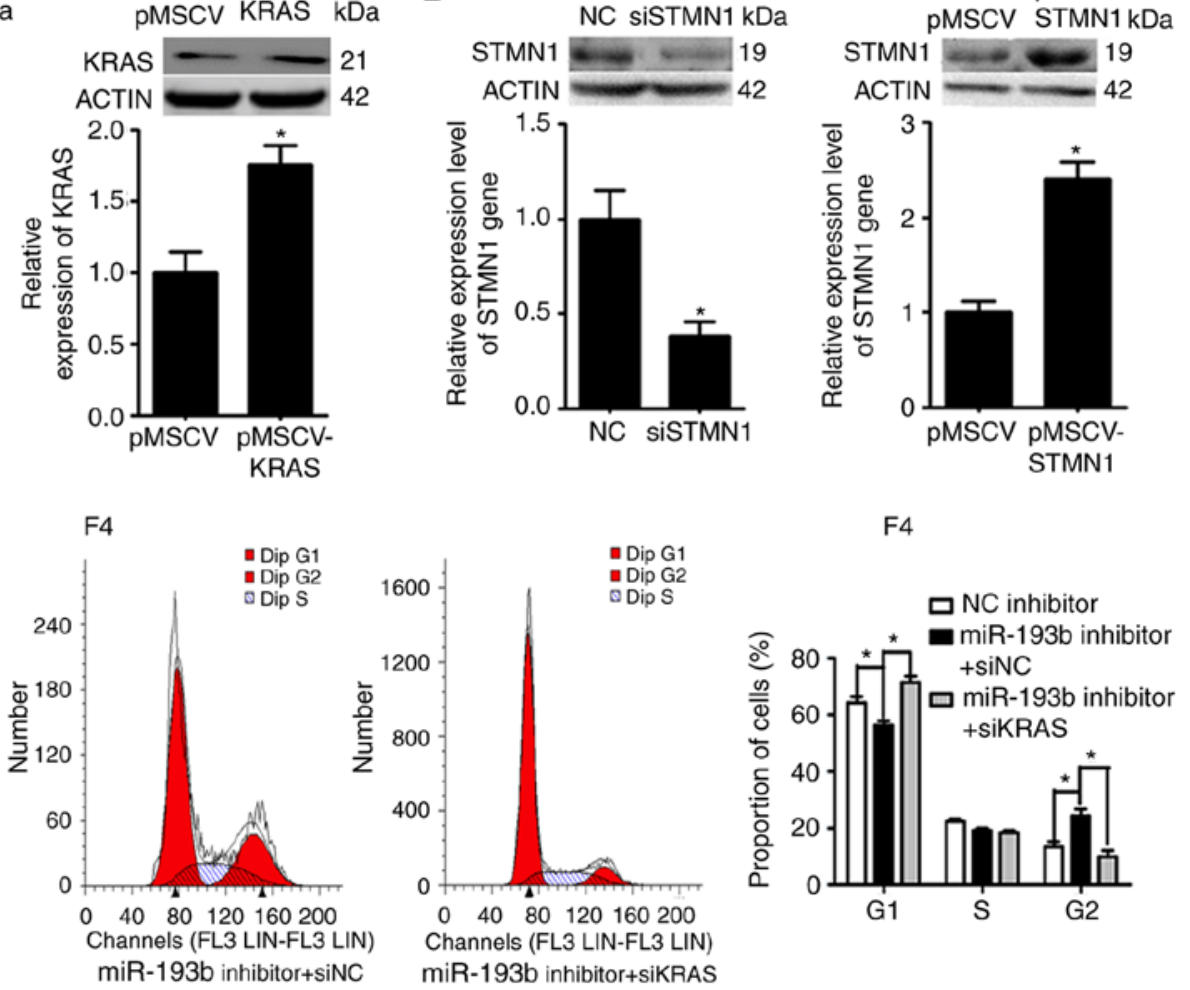

pMSCV-

PMSCV STMN1 kDa

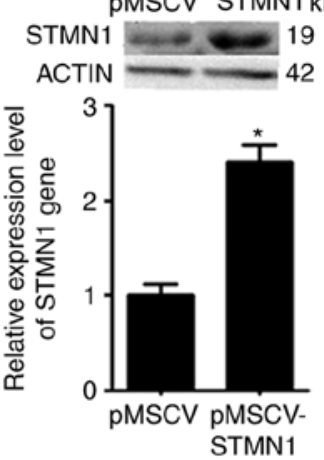

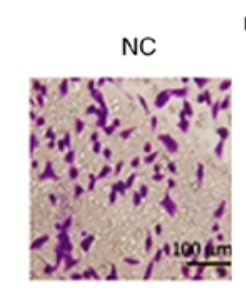

F5M2

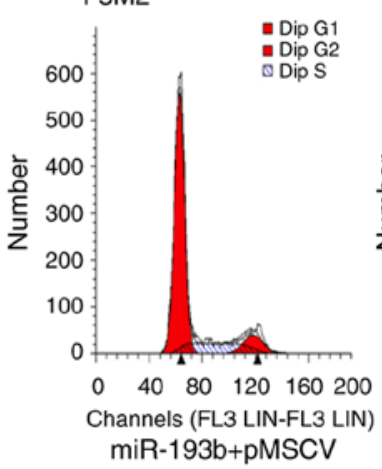

F4

R-193b inhibitor miR-193b inhibitor
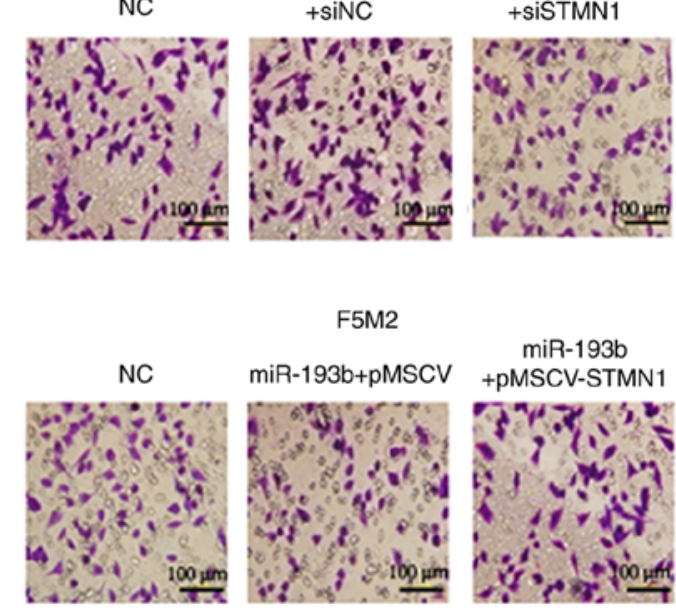

F5M2

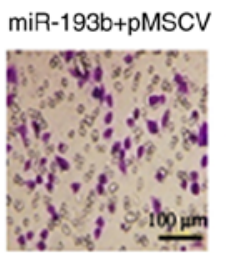

miR-193b

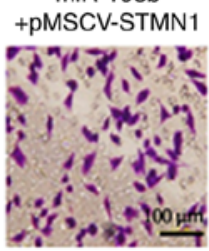

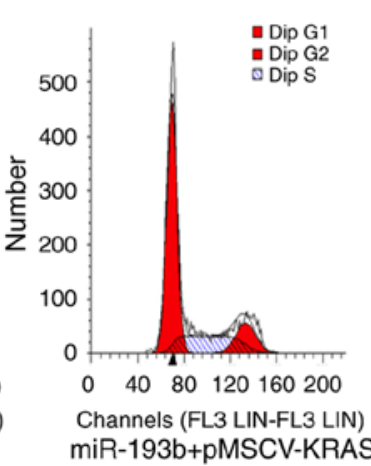

F5M2

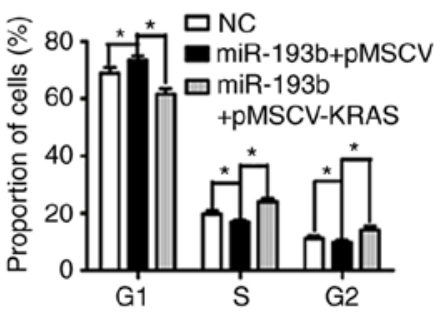

F4

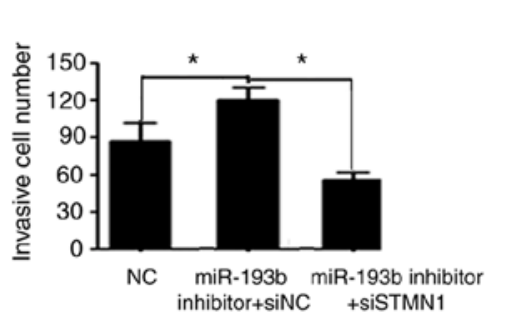

F5M2

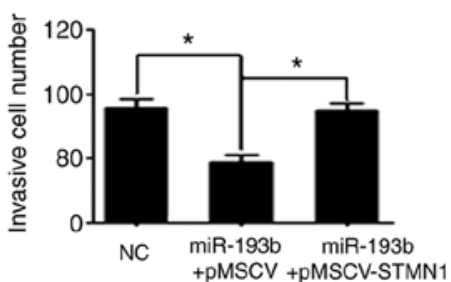

Figure 5. miR-193b inhibits proliferation via KRAS and suppresses migration and invasion via STMN1. (A) Transfection efficiency was verified after F4 and F5M2 cells were transfected with siKRAS or pMSCV-KRAS, respectively. (B) Transfection efficiency was verified after F4 and F5M2 cells were transfected with siSTMN1 or pMSCV-STMN1, respectively. (C) Co-transfection with pMSCV-KRAS reversed miR-193b-mimic induced cell cycle arrest at $\mathrm{G}_{1}$ phase in F5M2 cells, whereas co-transfection with siKRAS reversed the miR-193b inhibitor-induced increase in percentage of cells at $\mathrm{G}_{2}$ phase in F4 cells. (D) Transwell cell invasion assay (magnification $\mathrm{x} 40$ ). 

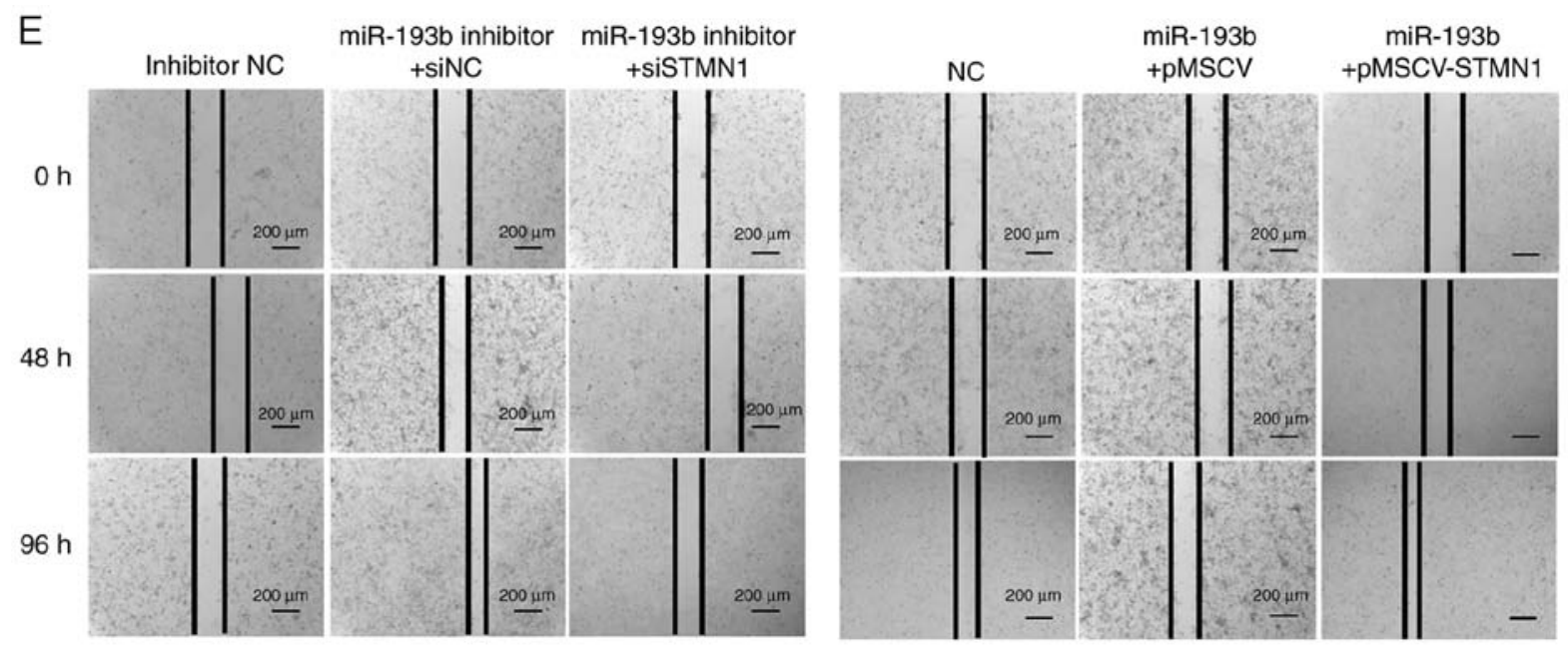

Figure 5. Continued. (E) wound-healing assay (magnification x20) revealed that co-transfection of F5M2 cells with pMSCV-STMN1 and miR-193b mimic or co-transfection of F4 cells with siSTMN1 and miR-193b inhibitor reversed the regulatory effects of miR-193b mimic or inhibitor. Data are presented as the mean \pm SD of at least three independent experiments. ${ }^{*} \mathrm{P}<0.05$. miR-193b, microRNA-193b; NC, negative control; STMN1, stathmin 1; si, small interfering RNA.

cells with the miR-193b inhibitor and siSTMN1 abolished miR-193b inhibitor-induced enhancement of tumor cell invasion (Fig. 5D). Similarly, wound-healing assay results revealed that pMSCV-STMN1 reversed miR-193b-induced inhibition of migration in F5M2 cells, whereas siSTMN1 abolished miR-193b inhibitor-induced enhancement of tumor cell migration in F4 cells (Fig. 5E).

MYC downregulates miR-193b expression. Using bioinformatics tools, it was predicted that the transcription factor MYC may bind to the promoter region of miR-193b and regulate its expression (Fig. 6A). Western blotting and RT-qPCR revealed that the expression levels of MYC were higher in F5M2 cells than in F4 cells (Fig. 6B). To validate the regulatory effects of MYC on miR-193b, the expression levels of miR-193b were examined after the expression of MYC was deliberately reduced in F5M2 cells (Fig. 6C) or enhanced in F4 cells (Fig. 6F). The expression of miR-193b was increased when MYC was downregulated in F5M2 cells (Fig. 6D). Conversely, miR-193b expression was decreased when MYC was overexpressed in F4 cells (Fig. 6G). In addition, the expression of KRAS, which is a target of miR-193b, was reduced after MYC was downregulated in F5M2 cells (Fig. 6E) and enhanced after MYC was upregulated in F4 cells (Fig. 6H).

MYC and miR-193b exert mutual suppressive effects against each other. The promoter reporter assay revealed that MYC could reduce the luciferase activity of the reporter construct harboring the wild-type promoter region of miR-193b (Fig. 7A). To further validate the binding between MYC and miR-193b, ChIP-qPCR was performed. In the ChIP-qPCR assay, six primers were designed according to the predicted binding sites on miR-193b. It was revealed that MYC was able to bind to the promoter region of miR-193b, and the relative enrichment levels of the precipitated DNA for anti-MYC groups were 1.5-5-fold greater than those for the IgG MOCK control groups (Fig. 7B).

Following overexpression of miR-193b in F5M2 cells, RT-qPCR and western blot analysis revealed that the expression of MYC was decreased (Fig. 7C). In contrast, the expression of MYC was increased after miR-193b expression was downregulated in F4 cells (Fig. 7D). Furthermore, when xenograft tumor sections were subjected to immunofluorescence staining for MYC, tumor sections from mice injected with F5M2 cells with upregulated miR-193b exhibited relatively lower MYC expression than those from mice in the control group. These results indicated that miR-193b had a negative effect on MYC expression. However, through exploring various target gene prediction websites, no direct binding site for miR-193b was detected on the 3'-UTR of MYC, indicating that miR-193b may negatively modulate the expression of MYC through some unknown mechanism. Since miR-193b has many direct targets, it is possible that one or several of these direct targets may subsequently influence the expression of MYC. Further research is required to clarify the mechanism underlying negative regulation of MYC by miR-193b.

\section{Discussion}

Due to their critical role in tumor generation and progression, miRNAs have been recognized as potential therapeutic targets for cancer treatment. By comparing the miRNA expression of two primary osteosarcoma cell lines with different metastatic potentials, miR-193b was identified as a potential regulator of malignant behaviors in osteosarcoma. Notably, miR-193b has been reported as a tumor suppressor that may be downregulated in various types of cancer, such as gastric cancer (24), Ewing sarcoma (25), colorectal cancer (26), pancreatic cancer (27), glioma (28), lung cancer (13), hepatocellular carcinoma (11), prostate cancer (10) and melanoma (29). Although the majority of reports have indicated that miR-193b may serve a protective role against various types of cancer, in head and neck squamous cell carcinoma, miR-193b functioned as a tumor promoter via the negative regulation of neurofibromin 1 (30). The underlying reason for this discrepancy remains unclear, but it might be due in part to organ-specific actions and the different cellular contexts of tumors (31). To the best of our knowledge, the role of miR-193b in the malignant behavior 
A

Predicted binding sites

(-982 bp cgctcCACGTGcgcccg-966 bp)

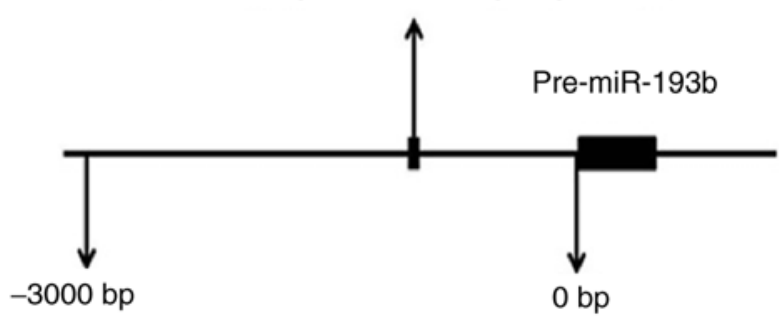

miR-193b promoter schematic diagram

C $\quad$ NC $\quad$ simYC1 1 simYC2
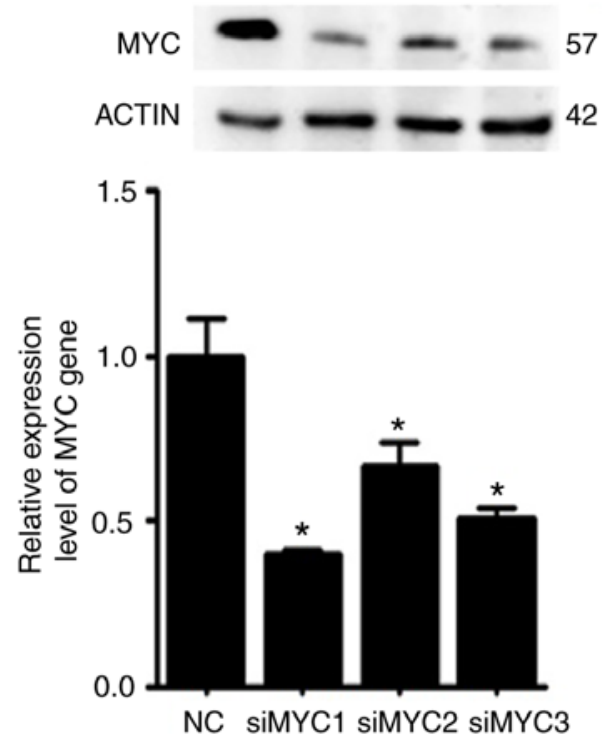

F

pBABE pBABE-MYC kDa
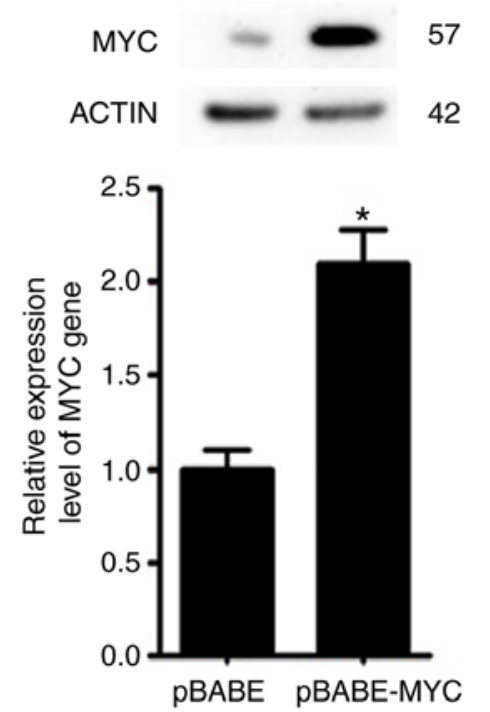

B
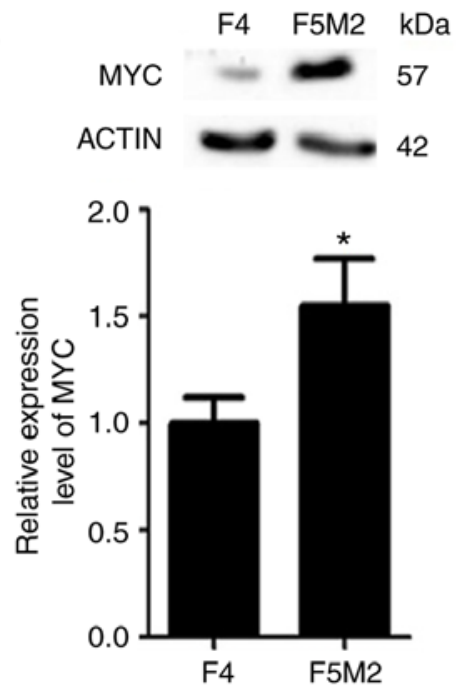

E NC siMYC1 siMYC2 siMYC3 kDa

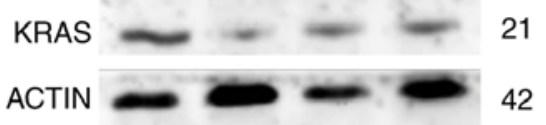

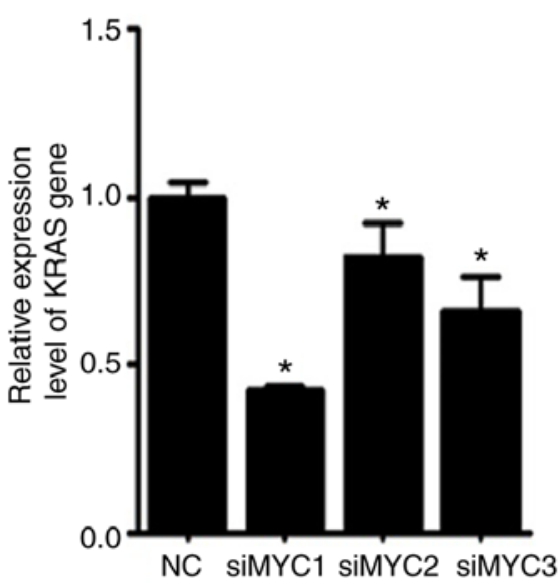

$\mathrm{H}$ pBABE pBABE-MYC kDa

G
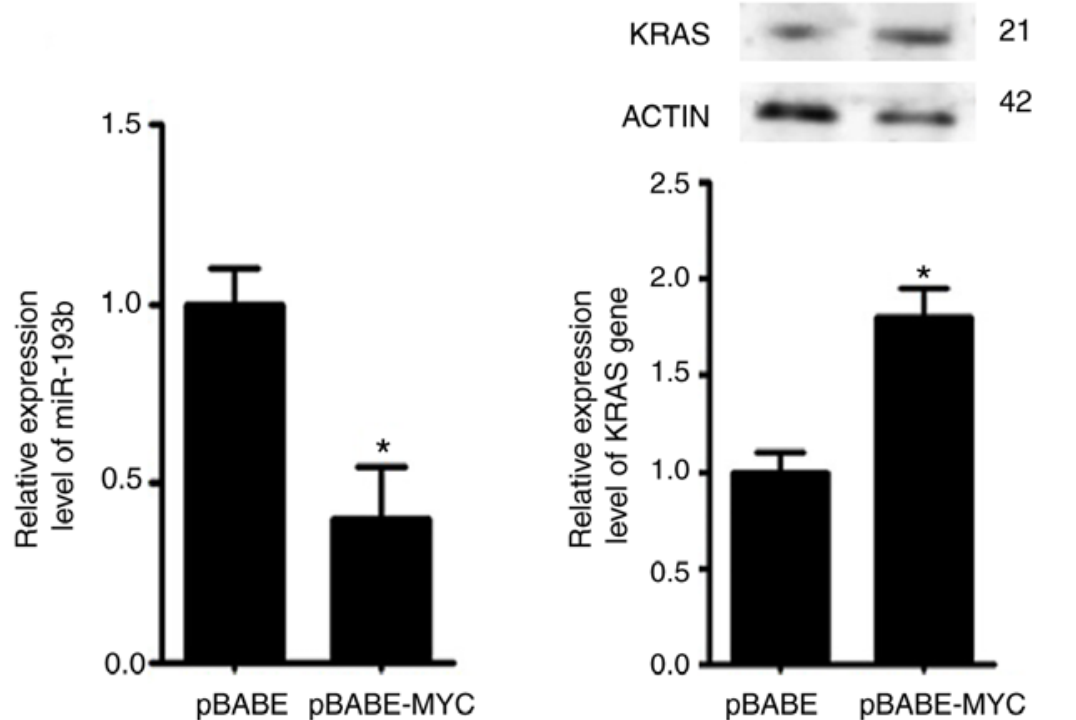
2
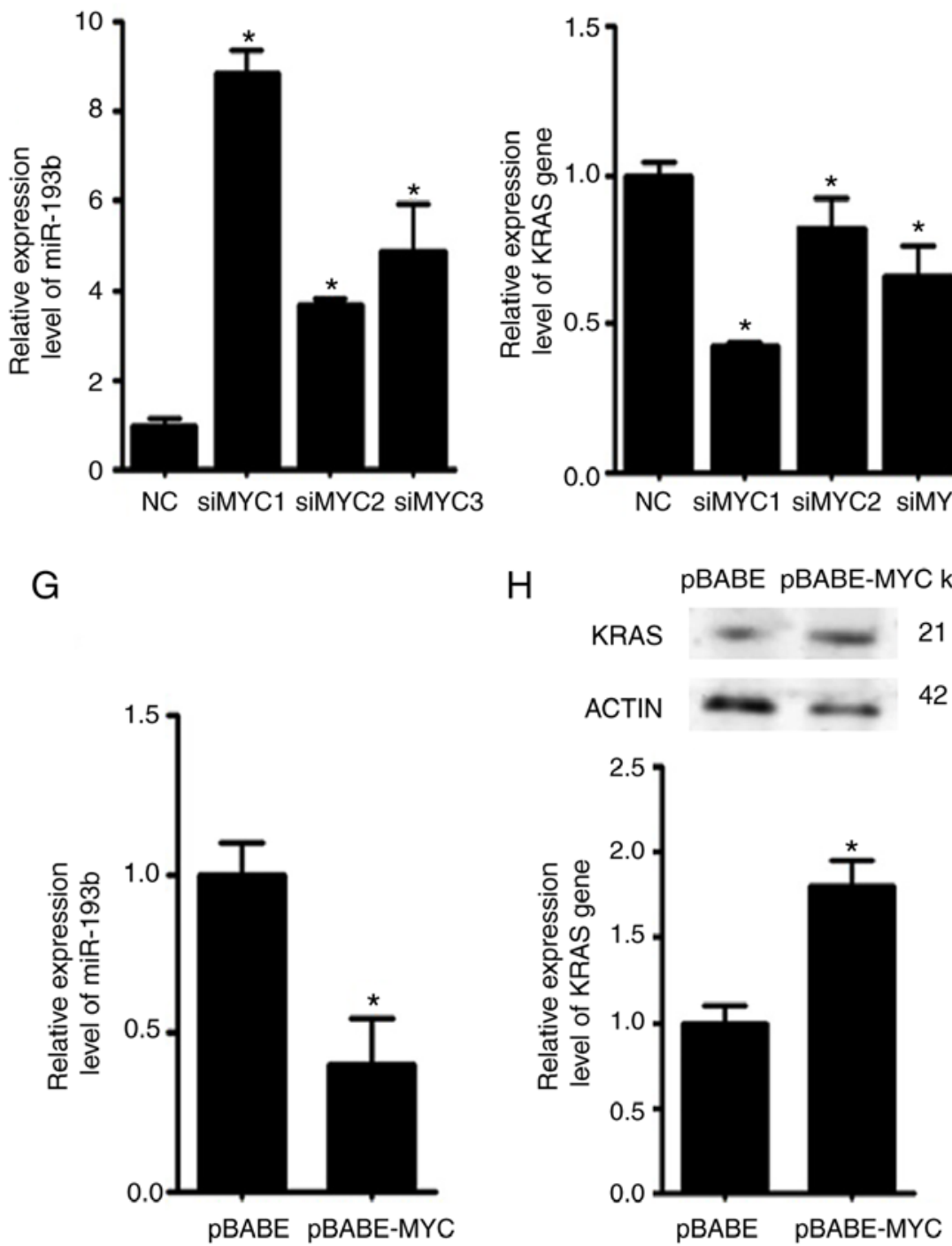

Figure 6. MYC exerts negative effects on miR-193b expression. (A) Schematic diagram showing the predicted binding site of MYC in the promoter region of miR-193b. (B) MYC expression was relatively higher in F5M2 cells compared with F4 cells. (C) Knockdown efficiency of MYC was verified in F5M2 cells. (D) Knockdown of MYC in F5M2 cells enhanced the expression of miR-193b and (E) subsequently reduced the expression of KRAS. (F) Overexpression of MYC in F4 cells (G) induced inhibition of miR-193b expression and (H) enhanced KRAS expression. Data are presented as the mean \pm SD of at least three independent experiments. " $\mathrm{P}<0.05$. miR-193b, microRNA-193b; NC, negative control; si, small interfering RNA. 
A

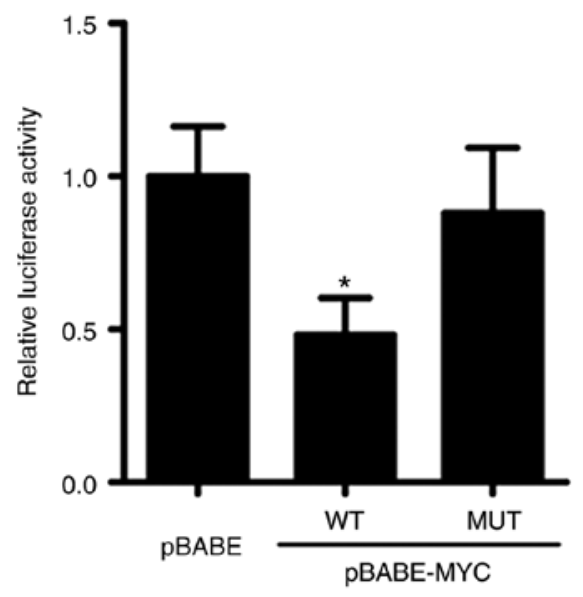

C
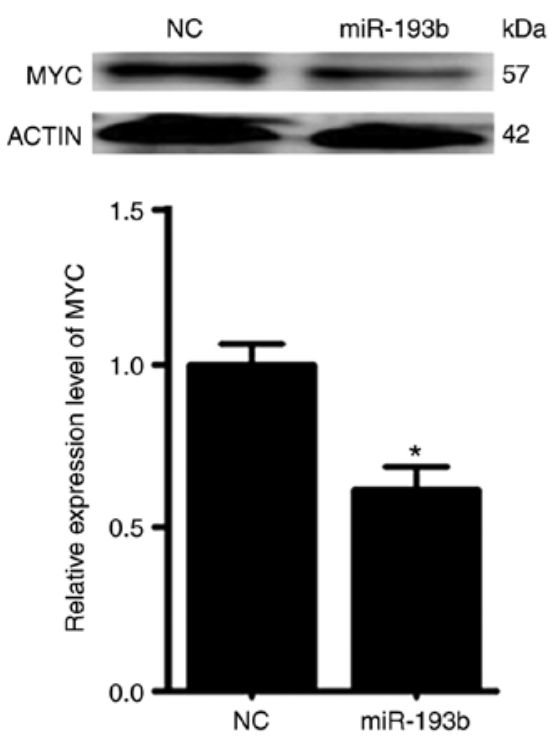

B
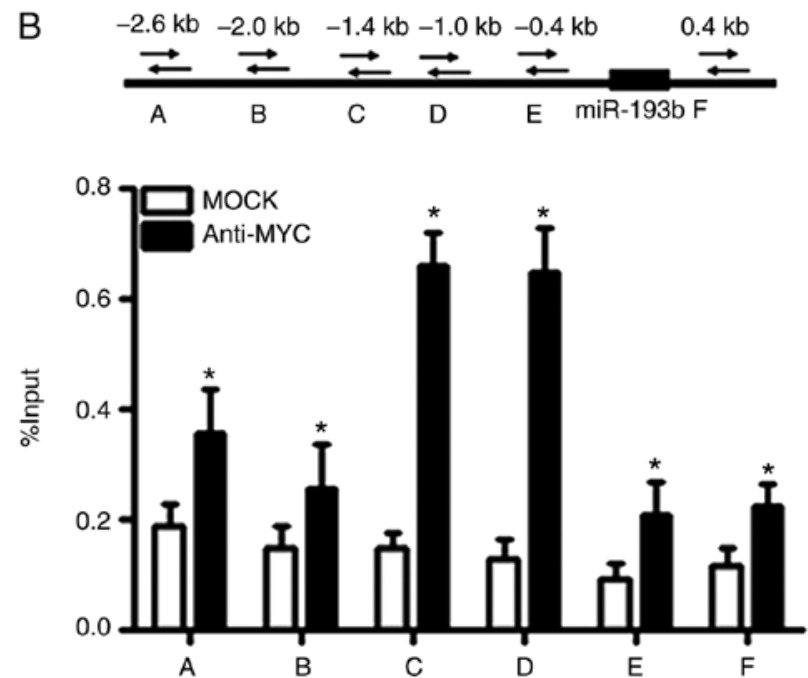

D

$\mathrm{E}$

DAPI

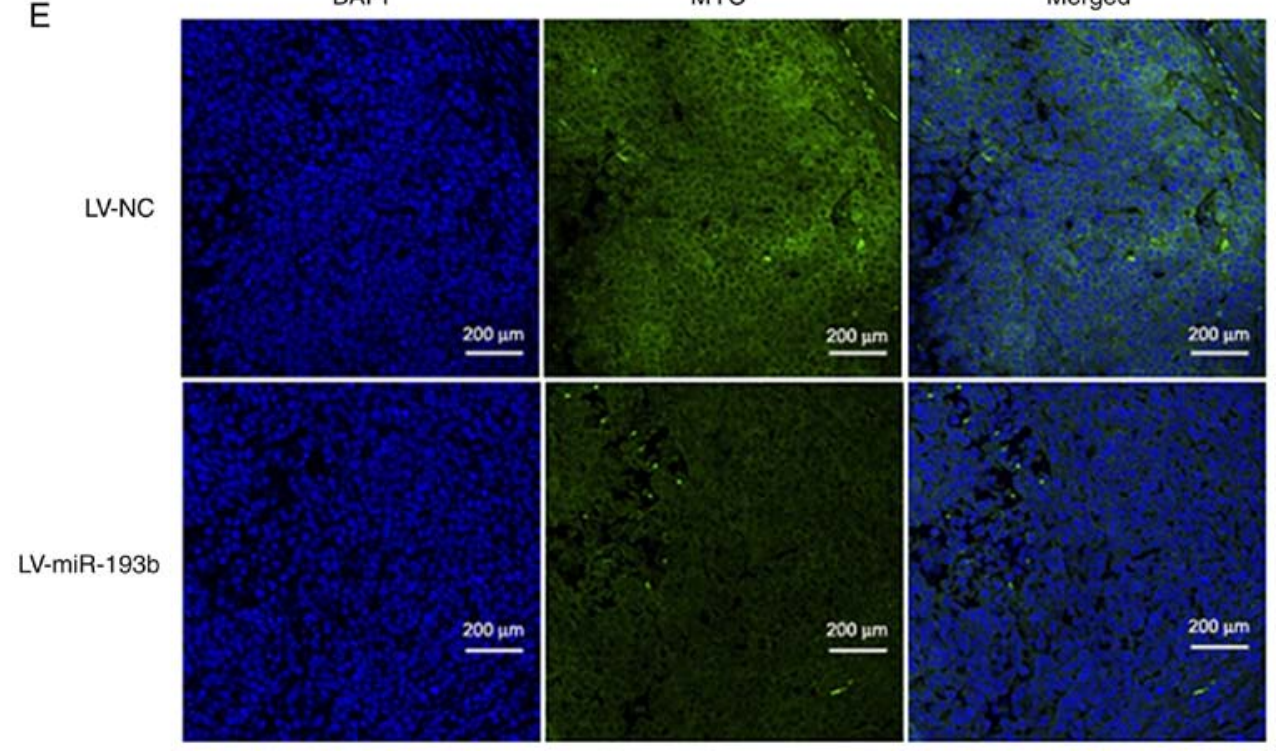

MYC

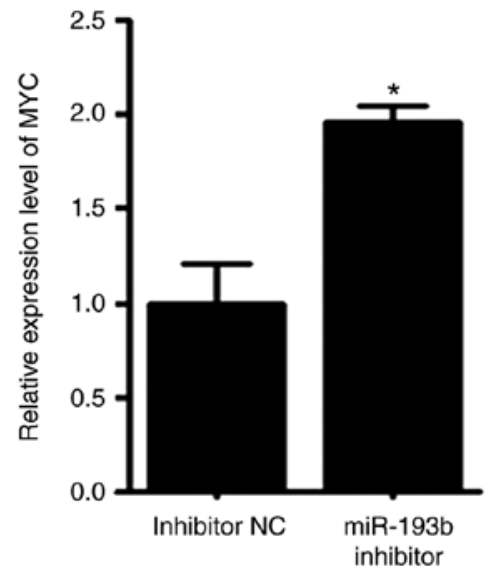

LV-miR-193b

LV-NC

Figure 7. miR-193b forms a negative feedback loop with MYC. (A) Promoter reporter assay and (B) chromatin immunoprecipitation-quantitative PCR assay revealed that MYC could directly bind to the promoter region of miR-193b and suppress the expression of miR-193b. (C) Transfection with a miR-193b mimic decreased MYC expression in F5M2 cells, (D) whereas transfection with a miR-193b inhibitor enhanced MYC expression in F4 cells. (E) Immunofluorescence staining of xenograft tumor tissue also indicated a negative influence of miR-193b on MYC expression. Data are presented as the mean \pm SD of at least three independent experiments. ${ }^{*} \mathrm{P}<0.05$. LV, lentivirus; miR-193b, microRNA-193b; NC, negative control. 
of osteosarcoma remains to be elucidated. Thus, a thorough investigation was performed in the present study to reveal the role of miR-193b in the generation and development of osteosarcoma.

Compared with the highly metastatic F5M2 cell line, F4 cells with a low metastatic capacity exhibited relatively high expression levels of miR-193b. This result indicated that, in accordance with the majority of published data, miR-193b may act as a tumor suppressor in osteosarcoma. To confirm this hypothesis, gain-and-loss-of-function studies were performed. As expected, upregulation of miR-193b in F5M2 cells significantly inhibited cell proliferation, cell cycle progression, cell migration and cell invasion, and markedly promoted apoptosis. Conversely, knockdown of miR-193b expression using inhibitors in F4 cells exerted the opposite effects. Furthermore, in vivo studies demonstrated that, compared with mice injected with wild-type F5M2 cells, nude mice injected with F5M2 cells with increased miR-193b levels exhibited reduced tumor mass and fewer lung metastatic nodules. Based on the aforementioned evidence, it was suggested that miR-193b may have a tumor-suppressive role in osteosarcoma.

Published studies have demonstrated that the tumor-suppressive role of miR-193b is mainly caused by inhibition of the expression of its target genes (9-13). To investigate the molecular mechanism underlying the tumor-suppressive role of miR-193b in osteosarcoma, numerous miRNA target gene prediction websites were used, and KRAS and STMN1 were identified as two potential targets for miR-193b. The expression levels of KRAS and STMN1 were further evaluated and it was revealed that they were negatively associated with the levels of miR-193b. The upregulation of miR-193b in F5M2 cells inhibited the expression of KRAS and STMN1, whereas the downregulation of miR-193b in F4 cells enhanced their expression, demonstrating negative influences of miR-193b on KRAS and STMN1 expression. To further verify whether miR-193b influenced the expression of KRAS and STMN1 by directly targeting their promoter regions, a luciferase reporter assay was performed. It was revealed that miR-193b reduced the luciferase activity of the reporter gene with the wild-type construct but not with the mutant 3'-UTR, demonstrating that miR-193b could indeed reduce KRAS and STMN1 expression by directly binding to their 3'-UTRs.

STMN1 encodes a cytosolic phosphoprotein, which is involved in the formation and function of the mitotic spindle. By acting as a microtubule destabilizer, STMN1 participates in various cellular biological processes, such as cell division, motility and differentiation (32). Recent studies have demonstrated that STMN1 may act as an oncogene, which was upregulated in numerous types of malignant tumors, including lung cancer (33), esophageal carcinoma (34), breast cancer (35), hepatocellular carcinoma (36), gastric cancer (37), pancreatic cancer (38) and colorectal cancer (39). STMN1 may promote the occurrence and development of tumors by interfering with microtubule dynamics, enhancing tumor cell proliferation and motility, and inhibiting apoptosis (40). The oncogenic role of STMN1 in osteosarcoma has also been revealed by recent publications. Jiang et al (41) reported that the inhibition of STMN1 expression using small interfering RNA induced growth inhibition, cell cycle arrest and apoptosis. Other researchers have reported that STMN1 may be involved in the drug resistance of osteosarcoma, and inhibition of STMN1 enhanced the chemosensitivity of osteosarcoma cells (42-44). In the present study, pMSCV-STMN1 reversed miR-193b-induced inhibition of migration and invasion in F5M2 cells, indicating that miR-193b may inhibit migration and invasion of osteosarcoma through STMN1.

The KRAS oncogene, which encodes a GTPase signaling protein, is a key driver of tumorigenesis (45). An oncogenic role of KRAS in osteosarcoma has been reported in recent years. Sun et al (46) investigated the differences in gene expression levels between metastatic and nonmetastatic osteosarcoma, and demonstrated that KRAS had the potential to be used as a biomarker for highly metastatic osteosarcoma. Zhang et al (47) reported that KRAS was a target of let-7a that may interfere with the viability, invasiveness and migration of osteosarcoma cells. Additionally, quercetin, a potential anticancer agent, enhanced the cisplatin sensitivity of osteosarcoma by modulating the miR-217-KRAS axis (48). In the present study, pMSCV-KRAS reversed miR-193b-induced cell cycle arrest at $G_{1}$ phase, indicating that miR-193b inhibited the proliferation of osteosarcoma cells through KRAS.

Althougha substantial number of publications have discussed the roles of miRNAs and their targets in human cancer, only a small number of them concern the upstream molecules that regulate miRNAs. MYC, which is a key basic helix-loop-helix leucine zipper transcription factor, acts as an important regulator of several cellular processes (49-51). Moreover, MYC has been recognized as an oncogene that is overexpressed in several types of human cancer $(52,53)$, including osteosarcoma (54). In recent years, increasing evidence has indicated that there is a mutual regulatory effect between miRNAs and MYC. This study investigated the involvement of MYC in the miRNA-regulated malignant behaviors of osteosarcoma cells. In contrast to miR-193b, highly metastatic F5M2 cells had a higher expression level of MYC than F4 cells with low metastatic potential. Furthermore, the downregulation of MYC in F5M2 cells significantly enhanced the expression of miR-193b and subsequently reduced the expression of KRAS. Conversely, the upregulation of MYC in F4 cells revealed opposite results. Taken together, these findings indicated that MYC may act as an upstream negative regulator of miR-193b. To test this hypothesis, several online databases were searched and it was revealed that a potential binding site for MYC is present in the promoter region of miR-193b. The direct binding of MYC to the miR-193b promoter region was validated using a ChIP-qPCR assay and luciferase reporter assay. Considering the recently published data regarding the mutual regulatory effects between MYC and miRNAs, this study aimed to assess whether the expression of MYC was subjected to regulation by miR-193b. Overexpression of miR-193b induced a reduction in MYC expression, whereas downregulation of miR-193b caused an increase in MYC expression. The negative regulation of MYC by miR-193b was also supported by in vivo studies, which revealed that MYC expression was relatively lower in tumor tissues from nude mice injected with miR-193b-upregulated F5M2 cells compared with the control group. To determine whether miR-193b regulated MYC through directly targeting the MYC 3'-UTR, various target gene prediction websites were explored. However, no direct binding site for miR-193b was identified. Since one miRNA can have a great number of targets, 
it may be hypothesized that miR-193b influences the expression of MYC indirectly through targeting other MYC-regulatory proteins or miRNAs.

In conclusion, the evidence from this study indicated that MYC and miR-193b exhibited mutual negative interactions with each other. Such a mutual interaction could lead to sustained MYC activation, miR-193b downregulation, STMN1 and KRAS upregulation, and may eventually promote development and metastasis of osteosarcoma.

\section{Acknowledgements}

Not applicable.

\section{Funding}

This study was supported by the Natural Science Foundation of China (grant nos. 30901784, 81172289 and 81472633).

\section{Availability of data and materials}

The datasets used and/or analyzed during the present study are available from the corresponding author on reasonable request.

\section{Authors' contribution}

LQS and TW designed the study and helped interpret the data. JJG performed the experiments, acquired the data and wrote the manuscript. FY, XC, WW, JPZ and YFL performed the experiments and acquired the data. SM performed data analysis, helped write the manuscript and revised the manuscript. All authors read and approved the final manuscript, and agreed to be accountable for all aspects of the research in ensuring that the accuracy or integrity of any part of the work are appropriately investigated and resolved.

\section{Ethic approval and consent to participate}

All animal handling and experimental procedures were approved by the Animal Ethics Committees of The Fourth Military Medical University and were in accordance with the guidelines of the China Council of Animal Care.

\section{Patient consent for publication}

Not applicable.

\section{Competing interest}

The authors declare that they have no competing interests.

\section{References}

1. Ottaviani G and Jaffe N: The epidemiology of osteosarcoma. Cancer Treat Res 152: 3-13, 2009.

2. Marina N, Gebhardt M, Teot L and Gorlick R: Biology and therapeutic advances for pediatric osteosarcoma. Oncologist 9: 422-441, 2004.

3. Esquela-Kerscher A and Slack FJ: Oncomirs-microRNAs with a role in cancer. Nat Rev Cancer 6: 259-269, 2006.

4. Bartel DP: MicroRNAs: Genomics, biogenesis, mechanism, and function. Cell 116: 281-297, 2004.
5. Gao J, Yang TT, Qiu XC, Yu B, Han JW, Fan QY and Ma BA: Cloning and identification of microRNA from human osteosarcoma cell line SOSP-9607. Ai Zheng 26: 561-565, 2007 (In Chinese).

6. Palmini G, Marini F and Brandi ML: What is new in the mirna world regarding osteosarcoma and chondrosarcoma? Molecules 7: 417, 2017.

7. Leichter AL, Sullivan MJ, Eccles MR and Chatterjee A: MicroRNA expression patterns and signalling pathways in the development and progression of childhood solid tumours. Mol Cancer 16: 15, 2017.

8. Chen X, Yang TT, Qiu XC, Ji ZG, Li CX, Long H, Zhou Y, Ma BA, Ma Q, Zhang X and Fan QY: Gene expression profiles of human osteosarcoma cell sublines with different pulmonary metastatic potentials. Cancer Biol Ther 11: 287-292, 2011.

9. Wu W, Lin Z, Zhuang Z and Liang X: Expression profile of mammalian microRNAs in endometrioid adenocarcinoma. Eur J Cancer Prev 18: 50-55, 2009.

10. Rauhala HE, Jalava SE, Isotalo J, Bracken H, Lehmusvaara S, Tammela TL, Oja $\mathrm{H}$ and Visakorpi T: MiR-193b is an epigenetically regulated putative tumor suppressor in prostate cancer. Int J Cancer 127: 1363-1372, 2010.

11. Xu C, Liu S, Fu H, Li S, Tie Y, Zhu J, Xing R, Jin Y, Sun Z and Zheng X: MicroRNA-193b regulates proliferation, migration and invasion in human hepatocellular carcinoma cells. Eur J Cancer 46: 2828-2836, 2010.

12. Li XF, Yan PJ and Shao ZM: Downregulation of miR-193b contributes to enhance urokinase-type plasminogen activator (uPA) expression and tumor progression and invasion in human breast cancer. Oncogene 28: 3937-3948, 2009.

13. Hu H, Li S, Liu J and Ni B: MicroRNA-193b modulates proliferation, migration, and invasion of non-small cell lung cancer cells. Acta Biochim Biophys Sin (Shanghai) 44: 424-430, 2012.

14. Jancik S, Drabek J, Radzioch D and Hajduch M: Clinical relevance of KRAS in human cancers. J Biomed Biotechnol 2010: 150960, 2010.

15. Mao Q, Chen Z, Wang K, Xu R, Lu H and He X: Prognostic role of high stathmin 1 expression in patients with solid tumors: Evidence from a meta-analysis. Cell Physiol Biochem 50: 66-78, 2018.

16. Zhao C, Li H, Wang L and Sun W: An immunohistochemical study of stathmin 1 expression in osteosarcoma shows an association with metastases and poor patient prognosis. Med Sci Monit 31: 6070-6078, 2018

17. Zhang H, He QY, Wang GC, Tong DK, Wang RK, Ding WB, Li C, Wei Q, Ding C, Liu PZ, et al: MiR-422a inhibits osteosarcoma proliferation by targeting BCL2L2 and KRAS. Biosci Rep 21: 38, 2018.

18. Swier L, Dzikiewicz-Krawczyk A, Winkle M, van den Berg A and Kluiver J: Intricate crosstalk between MYC and non-coding RNAs regulates hallmarks of cancer. Mol Oncol 13: 26-45, 2019.

19. Zhang N, Wang X, Huo Q, Sun M, Cai C, Liu Z, Hu G and Yang Q: MicroRNA-30a suppresses breast tumor growth and metastasis by targeting metadherin. Oncogene 33: 3119-3128, 2014.

20. Livak KJ and Schmittgen TD: Analysis of relative gene expression data using real-time quantitative PCR and the 2(-Delta Delta C(T)) method. Methods 25: 402-408, 2001.

21. Bai JX, Yan B, Zhao ZN, Xiao X, Qin WW, Zhang R, Jia LT, Meng YL, Jin BQ, Fan DM, et al: Tamoxifen represses miR-200 microRNAs and promotes epithelial-to-mesenchymal transition by up-regulating c-Myc in endometrial carcinoma cell lines. Endocrinology 154: 635-645, 2013.

22. Wang W, Xiao X, Chen X, Huo Y, Xi WJ, Lin ZF, Zhang D, Li YF, Yang F, Wen WH, et al: Tumor-suppressive miR-145 co-repressed by TCF4- $\beta$-catenin and PRC2 complexes forms double-negative regulation loops with its negative regulators in colorectal cancer. Int J Cancer 142: 308-321, 2018.

23. Wang YQ, Jiang DM, Hu SS, Zhao L, Wang L, Yang MH, Ai ML, Jiang HJ, Han Y, Ding YQ and Wang S: SATB2-AS1 suppresses colorectal carcinoma aggressiveness by inhibiting SATB2-Dependent snail transcription and epithelial-mesenchymal transition. Cancer Res 79: 3542-3556, 2019.

24. Wang L, Zhang Y, Zhao L, Liu S, Yu S, Ma Y and Sun G: MicroRNA-193b inhibits the proliferation, migration and invasion of gastric cancer cells via targeting cyclin D1. Acta Histochem 118: 323-330, 2016.

25. Moore C, Parrish JK and Jedlicka P: MiR-193b, downregulated in Ewing Sarcoma, targets the ErbB4 oncogene to inhibit anchorage-independent growth. PLoS One 12: e0178028, 2017.

26. GuoF,Luo Y,Mu YF, Qin SL, Qi Y, Qiu YE and Zhong M: MiR-193b directly targets STMN1 and inhibits the malignant phenotype in colorectal cancer. Am J Cancer Res 6: 2463-2475, 2016. 
27. Jin X, Sun Y, Yang H, Li J, Yu S, Chang X, Lu Z and Chen J: Deregulation of the MiR-193b-KRAS axis contributes to impaired cell growth in pancreatic cancer. PLoS One 10: e0125515, 2015.

28. Zhong Q, Wang T, Lu P, Zhang R, Zou J and Yuan S: MiR-193b promotes cell proliferation by targeting Smad3 in human glioma. J Neurosci Res 92: 619-626, 2014.

29. Chen J, Zhang X, Lentz C, Abi-Daoud M, Paré GC, Yang X, Feilotter HE and Tron VA: MiR-193b regulates Mcl-1 in melanoma. Am J Pathol 179: 2162-2168, 2011.

30. Lenarduzzi M, Hui AB, Alajez NM, Shi W, Williams J, Yue S, O'Sullivan B and Liu FF: MicroRNA-193b enhances tumor progression via down regulation of neurofibromin 1 . PLoS One 8: e53765, 2013.

31. Sampson VB, Yoo S, Kumar A, Vetter NS and Kolb EA: MicroRNAs and potential targets in osteosarcoma: Review. Front Pediatr 3: 69, 2015.

32. Rubin CI and Atweh GF: The role of stathmin in the regulation of the cell cycle. J Cell Biochem 93: 242-250, 2004.

33. Nie W, Xu MD, Gan L, Huang H, Xiu Q and Li B: Overexpression of stathmin 1 is a poor prognostic biomarker in non-small cel lung cancer. Lab Invest 95: 56-64, 2015.

34. Akhtar J, Wang Z, Jiang WP, Bi MM and Zhang ZP: Stathmin overexpression identifies high risk for lymphatic metastatic recurrence in $\mathrm{pN} 0$ esophageal squamous cell carcinoma patients. J Gastroenterol Hepatol 29: 944-950, 2014.

35. Baquero MT, Hanna JA, Neumeister V, Cheng H, Molinaro AM, Harris LN and Rimm DL: Stathmin expression and its relationship to microtubule-associated protein tau and outcome in breast cancer. Cancer 118: 4660-4669, 2012.

36. Chen YL, Uen YH, Li CF, Horng KC, Chen LR, Wu WR, Tseng HY, Huang HY, Wu LC and Shiue YL: The E2F transcription factor 1 transactives stathmin 1 in hepatocellular carcinoma. Ann Surg Oncol 20: 4041-4054, 2013.

37. Kang W, Tong JH, Chan AW, Lung RW, Chau SL, Wong QW, Wong N, Yu J, Cheng AS and To KF: Stathmin1 plays oncogenic role and is a target of microRNA-223 in gastric cancer. PLoS One 7: e33919, 2012

38. Lu Y, Liu C, Cheng H, Xu Y, Jiang J, Xu J, Long J, Liu L and $\mathrm{Yu} \mathrm{X}$ : Stathmin, interacting with $\mathrm{Nf}-\kappa \mathrm{B}$, promotes tumor growth and predicts poor prognosis of pancreatic cancer. Curr Mol Med 14: 328-339, 2014.

39. Tan HT, Wu W, Ng YZ, Zhang X, Yan B, Ong CW, Tan S, Salto-Tellez M, Hooi SC and Chung MC: Proteomic analysis of colorectal cancer metastasis: Stathmin-1 revealed as a player in cancer cell migration and prognostic marker. J Proteome Res 11: $1433-1445,2012$

40. BiaoxueR,Xiguang C,HuaLand Shuanying Y:Stathmin-Dependent molecular targeting therapy for malignant tumor: The latest 5 years discoveries and developments. J Transl Med 14: 279, 2016.

41. Zhang HZ, Wang Y, Gao P, Lin F, Liu L, Yu B, Ren JH, Zhao H and Wang R: Silencing stathmin gene expression by survivin promoter-driven siRNA vector to reverse malignant phenotype of tumor cells. Cancer Biol Ther 5: 1457-1461, 2006.
42. Wang Z, He R, Xia H, Wei Y and Wu S: Knockdown of STMN1 enhances osteosarcoma cell chemosensitivity through inhibition of autophagy. Oncol Lett 13: 3465-3470, 2017.

43. Feng T, Qiao G, Feng L, Qi W, Huang Y, Yao Y and Shen Z: Stathmin is key in reversion of doxorubicin resistance by arsenic trioxide in osteosarcoma cells. Mol Med Rep 10: 2985-2992, 2014.

44. Wang R, Dong K, Lin F, Wang X, Gao P, Wei SH, Cheng SY and Zhang HZ: Inhibiting proliferation and enhancing chemosensitivity to taxanes in osteosarcoma cells by RNA interference-mediated downregulation of stathmin expression. Mol Med 13: 567-575, 2007.

45. Karnoub AE and Weinberg RA: Ras oncogenes: Split personalities. Nat Rev Mol Cell Biol 9: 517-531, 2008.

46. Sun B, Wang F, Li M and Yang M: Identifications of genetic differences between metastatic and non-metastatic osteosarcoma samples based on bioinformatics analysis. Med Oncol 32: 153, 2015.

47. Zhang S, Hou C, Li G, Zhong Y, Zhang J, Guo X, Li B, Bi Z and Shao M: A single nucleotide polymorphism in the 3'-untranslated region of the KRAS gene disrupts the interaction with let-7a and enhances the metastatic potential of osteosarcoma cells. Int J Mol Med 38: 919-926, 2016.

48. Zhang X, Guo Q, Chen J and Chen Z: Quercetin enhances cisplatin sensitivity of human osteosarcoma cells by modulating microRNA-217-KRAS axis. Mol Cells 38: 638-642, 2015

49. Li Y,Liu H, Lai C, Du X, Su Z and Gao S: The Lin28/let-7a/c-Myc pathway plays a role in non-muscle invasive bladder cancer. Cell Tissue Res 354: 533-541, 2013.

50. Akinyeke T, Matsumura S, Wang X, Wu Y, Schalfer ED, Saxena A, Yan W, Logan SK and Li X: Metformin targets c-MYC oncogene to prevent prostate cancer. Carcinogenesis 34: 2823-2832, 2013.

51. Dueck AC, Reinholz MM, Geiger XJ, Tenner K, Ballman K, Jenkins RB, Riehle D, Chen B, McCullough AE, Davidson NE, et al: Impact of c-MYC protein expression on outcome of patients with early-stage HER $2+$ breast cancer treated with adjuvant trastuzumab NCCTG (alliance) N9831. Clin Cancer Res 19: 5798-5807, 2013.

52. Li K, Chen MK, Situ J, Huang WT, Su ZL, He D and Gao X: Role of co-expression of c-Myc, EZH2 and p27 in prognosis of prostate cancer patients after surgery. Chin Med J (Engl) 126: 82-87, 2013.

53. Liu Z, Jiang Y, Hou Y, Hu Y, Cao X, Tao Y, Xu C, Liu S, Wang S Wang L, et al: The IkB family member Bcl-3 stabilizes c-Myc in colorectal cancer. J Mol Cell Biol 5: 280-282, 2013.

54. Pello OM and Andres V: Role of C-MYC in tumor-associated macrophages and cancer progression. Oncoimmunology 2: e22984, 2013

This work is licensed under a Creative Commons Attribution-NonCommercial-NoDerivatives 4.0 International (CC BY-NC-ND 4.0) License. 